\title{
Nomenclator Generum et Familiarum Diplopodorum III. A list of the Genus-, Family-, and Ordinal-Group names proposed in the Class Diplopoda from 1 January 2000 - 31 December 2014
}

\section{Nomenclator Generum et Familiarum Diplopodorum III. Список названий групп родового, семейственного и отрядного ранга, предложкенных в классе Diplopoda с 1 января 2000 по 31 декабря 2014 года}

\author{
Rowland M. Shelley*, Sergei I. Golovatch** \\ Роуленд М. Шемли*, Сергей И. Головач**
}

\footnotetext{
* Research Laboratory, North Carolina State Museum of Natural Sciences, MSC \#1626, Raleigh, NC 27699-1626 USA. E-mail: rowland.shelley@naturalsciences.org

** Institute for Problems of Ecology and Evolution, Russian Academy of Sciences, Leninsky pr. 33, Moscow 119071 Russia. E-mail: sgolovatch@yandex.ru

** Институт проблем экологии и эволюции РАН, Ленинский пр-т, 33, Москва 119071 Россия.
}

KEY WORDS: millipede, taxonomy, nomenclature.

КЛЮЧЕВЫЕ СЛОВА: двупарноногие многоножки, таксономия, номенклатура.

ABSTRACT. A nomenclator of the genus-, family-, and ordinal-group names proposed in the class Diplopoda from 1 January 2000 until 31 December 2014 is compiled to encompass the last 15 years, following both the Nomenclator I [Jeekel, 1971], which covered the 200 years from the time of Linnaeus through 31 December 1957, and the Nomenclator II [Shelley et al., 2000], covering the period from 1 January 1958 until 31 December 1999. A number of inadvertent omissions from and corrections to the previous nomenclator are also introduced.

РЕЗЮМЕ. Составлен номенклатор названий групп родовового, семейственного и отдрядного ранга, предложенных в классе Diplopoda с 1 января 2000 по 31 декабря 2014 г., т.е. за последние 15 лет, в продолжение как Номенклатора I [Jeekel, 1971], который охватывал 200 лет со времени Линнея до 31 декабря 1957 г., так и Номенклатора II [Shelley et al., 2000], который покрыл период с 1 января 1958 по 31 декабрч 1999 г. Кроме того, включены непреднамеренные пропуски в предыдущем номенклаторе и дан ряд исправлений.

\section{Introduction}

Fifteen years have elapsed since Shelley et al. [2000] published the Nomenclator Diplopodorum II, covering the period from 1 January 1958 - 31 December 1999. This work supplemented the Nomenclator I [Jeekel, 1971], which covered the 200 years from the time of Linnaeus through 31 December 1957 and is the most important work ever published in diplopod taxonomy [Hoffman, 1980; Shelley, 2007]. During these 15 years, RMS has maintained a roster of newly proposed diplopod names for a third Nomenclator, and circumstances dictate that it now be published, this time in a more readily accessible professional journal instead of a less available book. Shelley et al. [2000] included new family-group statuses but did so incompletely, omitting around 50. They also inadvertently omitted the following six new ordinal- and family-group taxa: Cambalomminae Mauriès, 1977; Pseudonannolenida Mauriès, 1983; Macrochaetosomatinae Mršić, 1992; Chelodesmidea and Paradoxosomatidea, both Hoffman, 1967; and Dalodesmidea Hoffman, 1980. We incorporate these and several missed genus-group taxa here, but include new statuses only when a completely new family- or ordinal-group taxon by the same name was erected during the time period covered by this document. We only consider new family- and genus-group names for living taxa, not fossils. Consequently, Hexecontasoma, gen.n., and Hexacontasomatidae, fam.n., both by Hannibal [2000]; Anaxeodesmus, gen.n. [Wilson, 2005a]; Zostragrammus, gen.n., Zostrogrammidae, fam.n., and Zosterogrammida, ord.n., all by Wilson [2005b]; and several other fossil taxa described since 1999 are not included.

The latest systematic placements, as of 1 January 2015 , are employed whether we agree with them or not; similarly, we employ the overall hierarchical taxonomies published by Shelley [2003] and Shear [2011]. When categories like tribes are uncertain or not provided by authors, they are not listed. Modern geographical 
Table. Summary of replacement names proposed by Hüseyin Özdikmen. Таблица. Резюме замещающих названий, предложенных Hüseyin Özdikmen.

\begin{tabular}{|c|c|c|c|c|c|c|}
\hline Order/Family & $\begin{array}{l}\text { Senior } \\
\text { Homonym }\end{array}$ & $\begin{array}{l}\text { Junior } \\
\text { Homonym }\end{array}$ & $\begin{array}{l}\text { Replacement } \\
\text { Name } \\
\text { Proposed by } \\
\text { Others } \\
\end{array}$ & $\begin{array}{l}\text { Replacement } \\
\text { Name } \\
\text { Proposed by } \\
\text { H. Özdikmen }\end{array}$ & $\begin{array}{l}\text { Status of } \\
\text { Özdikmen's } \\
\text { Name }\end{array}$ & Remarks \\
\hline $\begin{array}{l}\text { Spirostreptida } \\
\text { Spirostreptidae }\end{array}$ & $\begin{array}{l}\text { Tomogonus } \\
\text { d'Orbigny, } \\
1904 \\
\text { (Coleoptera) }\end{array}$ & $\begin{array}{l}\text { Tomogonus } \\
\text { Demange, } \\
1971\end{array}$ & None & $\begin{array}{l}\text { Umbraticus } \\
\text { Özdikmen, } \\
2009\end{array}$ & Valid & \\
\hline $\begin{array}{l}\text { Chordeumatida } \\
\text { Kirkayakidae }\end{array}$ & $\begin{array}{l}\text { Altajella } \\
\text { Kulkov, } 1962 \\
\text { (Brachiopoda) }\end{array}$ & $\begin{array}{l}\text { Altajella } \\
\text { Gulička, } 1972\end{array}$ & None & $\begin{array}{l}\text { Kirkayakus } \\
\text { Özdikmen, } \\
2008\end{array}$ & Valid & $\begin{array}{l}\text { Altajellidae } \\
\text { Mikhaljova et } \\
\text { Golovatch, } 2000 \\
\text { is replaced by } \\
\text { Kirkayakidae } \\
\text { Özdikmen, } 2008\end{array}$ \\
\hline $\begin{array}{l}\text { Polydesmida } \\
\text { Paradoxosoma- } \\
\text { tidae }\end{array}$ & $\begin{array}{l}\text { Szechuanella } \\
\text { Lu, } 1959 \\
\text { (Trilobita) }\end{array}$ & $\begin{array}{l}\text { Szechuanella } \\
\text { Hoffman, } \\
1960\end{array}$ & $\begin{array}{l}\text { Sellanucheza } \\
\text { Enghoff, } \\
\text { Golovatch et } \\
\text { Nguyen, } 2004\end{array}$ & $\begin{array}{l}\text { Cemsunguria } \\
\text { Özdikmen, } \\
2007\end{array}$ & $\begin{array}{l}\text { Junior } \\
\text { objective } \\
\text { synonym, } \\
\text { INVALID }\end{array}$ & \\
\hline $\begin{array}{l}\text { Polydesmida } \\
\text { Chelodesmidae }\end{array}$ & $\begin{array}{l}\text { Cylindromus } \\
\text { Aurivillius, } \\
1891 \\
\text { (Coleoptera) }\end{array}$ & $\begin{array}{l}\text { Cylindromus } \\
\text { Loomis, } 1977\end{array}$ & None & $\begin{array}{l}\text { Delirus } \\
\text { Özdikmen, } \\
2009\end{array}$ & $\begin{array}{l}\text { Valid but } \\
\text { junior } \\
\text { subjective } \\
\text { synonym of } \\
\text { Ricodesmus } \\
\text { Chamberlin, } \\
1922\end{array}$ & $\begin{array}{l}\text { Cylindromus } \\
\text { Loomis, } 1977 \\
\text { was synonymi- } \\
\text { zed under Rico- } \\
\text { desmus Cham- } \\
\text { berlin, } 1922 \text {, by } \\
\text { Pérez-Asso } \\
\text { [2002], so Deli- } \\
\text { rus falls in the } \\
\text { same synonymy }\end{array}$ \\
\hline $\begin{array}{l}\text { Polydesmida } \\
\text { Paradoxosoma- } \\
\text { tidae }\end{array}$ & $\begin{array}{l}\text { Paratylopus } \\
\text { Matthew, } 1904 \\
\text { (Mammalia) }\end{array}$ & $\begin{array}{l}\text { Paratylopus } \\
\text { Korsós et } \\
\text { Golovatch, } \\
1989\end{array}$ & & $\begin{array}{l}\text { Lippus } \\
\text { Özdikmen, } \\
2009\end{array}$ & $\begin{array}{l}\text { Valid but } \\
\text { junior } \\
\text { subjective } \\
\text { synonym of } \\
\text { Tylopus } \\
\text { Jeekel, } 1968\end{array}$ & $\begin{array}{l}\text { Paratylopus } \\
\text { Korsós et } \\
\text { Golovatch, } 1989 \\
\text { was synony- } \\
\text { mized under } \\
\text { Tylopus Jeekel, } \\
\text { 1968, so Lippus } \\
\text { falls in the same } \\
\text { synonymy }\end{array}$ \\
\hline $\begin{array}{l}\text { Polydesmida } \\
\text { Chelodesmidae }\end{array}$ & $\begin{array}{l}\text { Curimagua } \\
\text { Forster et } \\
\text { Platnick, } 1976 \\
\text { (Araneae) }\end{array}$ & $\begin{array}{l}\text { Curimagua } \\
\text { Hoffman, } \\
1982\end{array}$ & $\begin{array}{l}\text { Curimaguana } \\
\text { Hoffman, } \\
2007\end{array}$ & $\begin{array}{l}\text { Vigilia } \\
\text { Özdikmen, } \\
2009\end{array}$ & $\begin{array}{l}\text { Junior } \\
\text { objective } \\
\text { synonym, } \\
\text { INVALID }\end{array}$ & \\
\hline
\end{tabular}

names, as of this date, are employed for countries, provinces/states, and smaller geopolitical subunits.

We also take this opportunity to correct errors in the Nomenclator II. The Fourth Edition of the International Code of Zoological Nomenclature, adopted on 1 January 2000, was in effect when this work was published, and new to it were articles 33.2.3.1 and 33.3.1, which were not in the Third Edition. According to these articles, the correct spelling of the type species of Ptyoiulus Cook, 1895, is pennsylvanicus, not pensylvanicus, as explained in the account under the order Julida. This was confirmed by Dr. P.K. Tubbs of the International Commission on Zoological Nomenclature in an email to RMS on 25 April 2001.

The following abbreviations are used through this work: Co. (County), Dept. (Department), Dist. (District), Edo. (Estado), Prov. (Province), Reg. (Region), USA (United States of America). Genera on which new family- or ordinal-group names have been based are denoted by asterisks $(*)$.

During the past 15 years, three diplopod nomenclatural notes were published by the controversial Turkish zoologist, Hüseyin Özdikmen [2007, 2008, 2009]. An entomologist, not a diplopodologist, Özdikmen also seems to search for zoological homonyms so as to validate replacement names, with himself as author, in the journal Munis Entomology and Zoology, where he himself is editor in chief! He proposed six new genusand one new family-group names to replace perceived junior homonyms, but older replacement names had already been established for two. Consequently, two Özdikmen names constitute rare objective synonyms of prior replacement names and unnecessarily confuse and complicate diplopod nomenclature; furthermore, the author of both junior homonyms, the eminent R.L. Hoffman, was alive at these times. According to Criter- 
ion 3 in the Code of Ethics ( $4^{\text {th }}$ edition, International Code of Zoological Nomenclature [2000: 124, 261]), which governed nomenclatural actions then, "A zoologist should not publish a new replacement name... for a junior homonym when the author of the latter is still alive; that author should be informed of the homonymy and be allowed a reasonable time...in which to establish a substitute name." Additionally, criterion 6 states, "Editors and others responsible for the publication of zoological papers should avoid publishing any material which appears to them to contain a breach of the above principles." Had Özdikmen followed criterion 3, Dr. Hoffman would have informed him that replacement names had already been proposed and his subsequent objective synonyms would have been avoided, and since he was also editor of the journal in which he published his own names, Özdikmen could have also avoided his unjustified actions by following criterion 6 , and he apparently did not do that either. Consequently, in addition to not, or inadequately, researching both situations, Özdikmen also violated both of these ethical criteria in wrongfully erecting both objective synonyms. For the benefit of future diplopodologists, we encapsulate Özdikmen's names and their statuses in the Table. To our knowledge, he has not published further diplopod replacement names, perhaps indicating that no more junior generic homonyms exist in our science.

\section{Errata in the Nomenclator II}

Cynotelopus. Erroneously placed in the family Zephroniidae, subfamily Zephroniinae (= Sphaeropoeidae, Sphaeropoeinae) [Shelley et al., 2000: 12], its correct placement is in the family Cyliosomatidae [Wesener, 2014]. Thanks to R. Mesibov for pointing out this error.

Principestreptus. Erroneously stated as deriving from Brazil [Shelley et al., 2000: 36], but actually coming from the Republic of São Tomé \& Principe, Gulf of Guinea, Atlantic Ocean. Thanks to J.-P. Mauriès for pointing out this error.

Baskoiulus. Erroneously stated to be an unavailable name [Shelley et al., 2000: 44], but actually validated by Mauriès, 1982. Bulletin du Muséum national d'Histoire naturelle, $4^{\text {ème }}$ sér., Section A, Nos 3-4, 4: 440, as a subgenus of Mesoiulus, with B. stammeri Verhoeff, 1938, designated as typespecies. Thanks to J.-P. Mauriès for pointing out this error.

Euzkadiulus. Erroneously stated as deriving from Spain [Shelley et al., 2000: 46], but actually coming from France. Thanks to J.-P. Mauriès for pointing out this error.

Occitaniulus. Erroneously stated as deriving from Spain [Shelley et al., 2000: 49], but acrtually coming from France. Thanks to J.-P. Mauriès for pointing out this error.

Phyllomatoiulus. A junior subjective synonym of Tachypodoiulus, first proposed not by Hoffman [1980], as erroneously stated by Shelley et al. [2000: 51], but by Mauriès, 1975. Bulletin de la Société d'Histoire Naturelle de Toulouse, 111(1-2): 129. Thanks to J.-P. Mauriès for pointing out this error.
Dorasoma. Erroneously stated to be an unavailable name [Shelley et al., 2000: 67], but actually validated by PedroliChristen \& Mauriès [1992: 467], with D. serratum Verhoeff, 1932, designated as type-species. Presently considered a junior subjective synonym of Bomogona (Ornithogona) helvetica (Verhoeff, 1894) [Pedroli-Christen, Mauriès, 1992]. Thanks to J.-P. Mauriès for pointing out this error.

Karukeromus. Erroneously stated to have been published in 1980 [Shelley et al., 2000: 109], but the actual publication date was 1981. Thanks to J.-P. Mauriès for pointing out this error.

\section{PENICILLATA: POLYXENIDA}

Afraustraloxenodes Nguyen Duy-Jacquemin, 2003. African Invertebrates, 44(1): 72-73. M. (By analogy, as Macroxenodes Silvestri, 1948, is masculine [Jeekel, 1971]).

Type-species. - A. namibiensis Nguyen Duy-Jacquemin, 2003. African Invertebrates, 44(1): 73-78; Namibia, towards Rehoboth and $150 \mathrm{~km}(83.7 \mathrm{mi})$ west of Windhoek; original designation. 4 species.

Polyxenidae, Polyxenoidea.

Condexenus Nguyen Duy-Jacquemin, 2006. Norwegian Journal of Entomology, 53: 238-239. M.

Type-species. - C. biramipalpus Nguyen Duy-Jacquemin, 2006. Norwegian Journal of Entomology, 53: 239243; Namibia, 23-24 km (14.4-15 mi) NW Keetmanshoop; original designation. 1 species.

Synxenidae, Synxenoidea.

CHILOGNATHA: PENTAZONIA: LIMACOMORPHA: GLOMERIDESMIDA

No new genus- or family-group taxa were proposed in Glomeridesmida from 2000-2014.

\section{ONISCOMORPHA: GLOMERIDA}

Cantabromeris Mauriès, 2005. Arthropoda Selecta, 14(3): 243. F

Type-species. - Protoglomeris cantabrica Mauriès et Vicente, 1977. Bulletin du Muséum National d'Histoire Naturelle, 3e Série, no. 452, Zoologie, 315: 543-545. Spain, Santander Prov., vicinity of Santander (town); original designation. 1 species.

Onychoglomerini, Glomerinae, Glomeridae.

*Mauriesia Golovatch, Mikhaljova et Chang, 2010. Zootaxa, 2477: 2-3. F.

Type-species. - M. splendida Golovatch, Mikhaljova et Chang, 2010. Zootaxa, 2477: 5-6. Taiwan, Nantou Co., Lugu Township, Sitou; original designation. 1 species.

Mauriesiinae, Glomeridae.

New family-group name: Mauriesiinae Golovatch, Mikhaljova et Chang, 2010. Zootaxa, 2477: 4-5. Familygroup component: Mauriesia Golovatch, Mikhaljova et Chang, 2010.

Nearctomeris Wesener, 2012. Zootaxa, 3258: 62. F.

Type-species. - N. inexpectata Wesener, 2012. Zootaxa, 3258: 63-65. USA, Alabama, Madison Co., Huntsville, Monte Sano State Park; original designation. 1 species.

Glomerini/Doderiini, Glomerinae, Glomeridae. 
Tectosphaera Mauriès, 2006. Arthropoda Selecta, 14(3): 243. F.

Type-species. - T. vicenteae Mauriès, 2006. Arthropoda Selecta, 14(3): 243-248. Spain, Galicia, near Orense, Beade; original designation. 1 species.

Remark: The printed date is 2005 , but the actual publication date was March 2006 (http://kmkjournals.com/journals/AS).

Doderiini, Glomerinae, Glomeridae.

\section{SPHAEROTHERIIDA}

Microsphaerotherium Wesener et VandenSpiegel, 2007. Journal of Afrotropical Zoology, 3: 154. N.

Type-species. - M. ivohibiensis (recte: ivohibiense) Wesener et VandenSpiegel, 2007. Journal of Afrotropical Zoology, 3: 154-160; Madagascar, Fianarantsoa, Réserve Spéciale de Ivohibe; original designation. 2 species [Wesener, 2009].

Arthrosphaeridae.

*Procyliosoma Silvestri, 1917. Bollettino del Laboratorio di Zoologia Generale e Agraria della Reale Scuola Superiore d'Agricoltura in Portici, 12: 75. N.

Type-species. - P. leae Silvestri, 1917. Bollettino del Laboratorio di Zoologia Generale e Agraria della Reale Scuola Superiore d'Agricoltura in Portici, 12: 77. Australia, Tasmania; original designation. 11 species.

Procyliosomatidae.

New family-group name: Procyliosomatidae Wesener, 2009. Cladistics, 25: 561-562.

Family-group component: Procyliosoma Silvestri, 1917. 68. F.

Tigridosphaera Jeekel, 2000. Myriapod Memoranda, 2:

Type-species. - Sphaeropoeus globus-magicus (recte: globusmagicus) Jeekel, 1951. Tijdschrift voor Entomologie, 93: 101. Malaysia, West Malaysia, Selangor, Bukit Lebong Forest Reserve; original designation. 4 species.

Sphaeropoeini, Zephroniinae, Zephroniidae.

HELMINTHOMORPHA: COLOBOGNATHA: POLYZONIIDA, PLATYDESMIDA, SIPHONOCRYPTIDA

No new genus- or family-group taxa were proposed in Polyzoniida, Platydesmida, and Siphonocryptida from 2000 2014.

\section{SIPHONOPHORIDA} 50. N.

Lomboknium Jeekel, 2001. Myriapod Memoranda, 3:

Type-species. - Siphonophora zehntneri Carl, 1912. Revue Suisse de Zoologie, 20: 510. Indonesia, Java; original designation. 3 species.

Remark: This action effectively constitutes validation of Lomboknium Verhoeff, 1941, which was invalidly proposed because no type species was designated.

Siphonophoridae.

HELMINTHOMORPHA: EUGNATHA: JULIFORMIA: JULIDA

Arvechamboides Shelley, 2007. Zootaxa, 1440: 62-63. M. Type-species. - A. ocala Shelley, 2007. Zootaxa, 1440: 63-67. USA, Florida, Marion Co., Ocala National Forest ca. $34.1 \mathrm{~km}$ (21.3 mi) NE Ocala; original designation. 1 species.
Pseudojulina, Aniulini, Parajulinae, Parajulidae, Parajuloidea.

Enghophyllum Lazányi et Vagalinski, 2013. European Journal of Taxonomy, 70: 2-4. N.

Type-species. - Brachyiulus (Chromatoiulus) naxius Verhoeff, 1901. Archiv für Naturgeschichte, 67(1): 98-99. Greece, Cyclades, Naxos Island; original designation. 2 species.

Brachyiulini, Julidae, Juloidea.

Mammamia Akkari, Stoev et Enghoff, 2011. ZooKeys, 114: 2. F.

Type-species. - M. profuga Akkari, Stoev et Enghoff, 2011. ZooKeys, 114: 3-5. Italy, Taranto, Grotta della Cava; monotypy. 1 species.

Brachyiulini, Julidae, Juloidea. 40. F.

*Nesoressa Shelley et Medrano, 2006. Zootaxa, 1285:

Type-species. - N. crawfordi Shelley et Medrano, 2006. Zootaxa, 1285: 40-48. USA, New Mexico, Socorro Co., $35.2 \mathrm{~km}$ (22 mi) SW Magdalena, summit of Mt. Withington in San Mateo Mountains; original designation. 1 species.

Nesoressini, Parajulinae, Parajulidae, Parajuloidea.

New family-group name: Nesoressini Shelley et Medrano, 2006. Zootaxa, 1285: 33-36. Family-group component: Nesoressa Shelley et Medrano, 2006.

*Pseudojulus Bollman, 1887. Entomologica Americana, 2: 227. M

Type-species. - Parajulus (Pseudojulus) obtectus Bollman, 1887. Entomologica Americana, 2: 227. USA, Florida, Escambia Co., Pensacola; subsequent designation, later the same year, by Bollman [1887: 38]. 5 species.

Remark: Proposed as a subgenus of Parajulus Humbert et DeSaussure, 1869; elevated to full generic status by Cook [1895: 6].

Pseudojulina, Aniulini, Parajulinae, Parajuliudae, Parajuloidea.

New family-group name: Pseudojulina Shelley, 2007. Zootaxa, 1541: 4. Family-group components: Pseudojulus Bollman, 1887; Arvechambus Causey, 1963; Arvechamboides Shelley, 2007.

Ptyoiulus Cook, in: Cook et Collins, 1895. Annals of the New York Academy of Sciences, 9: 6. M

Type-species. - Julus (Julus) pensylvanicus (recte: pennsylvanicus) Brandt, 1841. Bulletin Scientifique publié par l'Académie Impériale des Sciences de St.-Pétersbourg, 8: 102. USA, Pennsylvania; original designation. 3 species.

Remarks: Brandt [1841] spelled the species name and state with one "n", a spelling also used by Gervais [1847: 177]; Wood [1865: 201] corrected it to "pennsylvanicus" without comment and also spelled the state correctly. To the best of our knowledge, this " $2 \mathrm{n}$ " spelling has been used for 150 years by every subsequent author and always attributed to Brandt [1841], to the point that Hoffman [1999: 163] stated such. Under the previous, Third Edition, of the Code, this change would have been considered an unjustified emendation and rejected, but the Fourth Edition that became effective on 1 January 2000 allows such changes in certain circumstances. Article 33.2.3.1 of this edition states "when an unjustified emendation is in prevailing usage and is attributed to the original author and date it is deemed to be a justified emendation," and article 33.3.1 states "when an incorrect subsequent spelling is in prevailing usage and is 
attributed to the publication of the original spelling, the subsequent spelling and attribution are to be preserved and the spelling is deemed to be a correct original spelling." Consequently, "pennsylvanicus" is the correct original spelling and a justified emendation (P.K. Tubbs, in litt. to RMS on 25 April 2001).

Ptyoiulini, Ptyoiulinae, Parajulidae, Parajuloidea.

Titanophyllum Akkari, Stoev et Enghoff, 2011. ZooKeys, 114: 5. N.

Type-species. - T. spiliarum Akkari, Stoev et Enghoff, 2011. ZooKeys, 114: 5-8. Greece, Magnesia, Othris Mountains, Kofi, Titanospilia (Cave of Titans); monotypy. 1 species.

Brachyiulini, Julidae, Juloidea.

\section{SPIROBOLIDA}

Alluviobolus Wesener, in: Wesener, Enghoff et Sierwald, 2009. ZooKeys, 19: 77-82. M.

Type-species. - A. laticlavius Wesener, in: Wesener, Enghoff et Sierwald, 2009. ZooKeys, 19: 82-83. Madagascar, Toliara Prov., Petriky; original designation. 3 species.

Pachybolidae, Trigoniulidea. M

Australocricus Jeekel, 2001. Myriapod Memoranda, 4:

Type-species. - Rhinocricus sennae Silvestri, 1898. Bollettino della Società Entomologica Italiana, 29: 230. Australia, Queensland, Cairns; original designation. 7 species.

Rhinocricidae, Rhinocricidea.

Austrostrophus Hoffman, 2003. Records of the Western Australian Museum, 22: 17. M.

Type-species. - A. stictopygus Hoffman, 2003. Records of the Western Australian Museum, 22: 17-21. Australia, Western Australia, Burrup Peninsula, Rocky Hill at Hearson Cove; original designation. 1 species.

Pachybolidae, Trigoniulidea.

*Aztecolus Chamberlin, 1943. Bulletin of the University of Utah, 34(6)[Biological Series, 8(2)]: 28. M.

Type-species. - Spirobolus nigrior Chamberlin, 1941. Entomological News, 52: 253-254. Mexico, Nuevo León, Villa Santiago (Hacienda Vista Hermosa - Horsetail Falls); original designation. 3 species.

Aztecolini, Spirobolinae, Spirobolidae, Spirobolidea.

New family-group name: Aztecolini Shelley, 2014. Insecta Mundi, 0357: 26-27. Family-group components: $A z$ tecolus Chamberlin, 1943; Chicobolus Chamberlin, 1947.

Bahorucocricus Pérez-Asso, 2004. Solenodon, 4: 16. M. Type-species. - B. crassus Pérez-Asso, 2004. Solenodon, 4: 16-17. Dominican Republic, Barahona Prov., Sierra de Bahoruco Oriental, between San Rafael and La Ciénega; monotypy. 1 species.

Rhinocricidae, Rhinocricidea.

Caprobolus Wesener, in: Wesener, Enghoff et Sierwald, 2009. ZooKeys, 19: 72-73. M.

Type-species. - C. andringitra Wesener, in: Wesener, Enghoff et Sierwald, 2009. ZooKeys, 19: 76-77. Madagascar, Fianarantsoa Prov., Andringitra Reserve along Volotsangana River, 40 km (25 mi) S Ambalavao; original designation. 1 species.

Pachybolidae, Trigoniulidea.
Carlocricus Jeekel, 2001. Myriapod Memoranda, 4: 89. M. Type-species. - Rhinocricus elberti Carl, 1912. Zoologische Jahrbücher, Abtheilung für Systematik, Geographie und Biologie der Thiere, 32: 170-171. Indonesia, Lesser Sunda Islands, Lombok, Sadang; Swela, Sembalum; Sumbawa, Batoe Doelang; original designation. 1 species.

Rhinocricidae, Rhinocricidea.

Colossobolus Wesener, in: Wesener, Enghoff et Sierwald, 2009. ZooKeys, 19: 17-24. M.

Type-species. - C. semicyclus Wesener, in: Wesener, Enghoff et Sierwald, 2009. ZooKeys, 19: 25-26. Madagascar, Antsiranana Prov., Forêt d'Antsahabe, 11.4 km (7.1 mi) W Daraina; original designation. 7 species.

Pachybolidae, Trigoniulidea.

Corallobolus Wesener, in: Wesener, Enghoff et Sierwald, 2009. ZooKeys, 19: 7-8. M.

Type-species. - C. cruentus Wesener, in: Wesener, Enghoff et Sierwald, 2009. ZooKeys, 19: 8-12. Madagascar, Toliara Prov., RNI Andohahela, Parcel 1, camp 3; original designation. 1 species.

Pachybolidae, Trigoniulidea.

Crurifarcimen Enghoff, 2011. Zootaxa, 2753: 8. N. (By analogy, as "farcimen" in Latin is neuter).

Type-species. - C. vagans Enghoff, 2011. Zootaxa, 2753: 8-16. Tanzania, Tanga Reg., East Usambara Mountains, Amani, forest clearing at Kwankoro Rd.; original designation. 1 species.

Pachybolidae, Trigoniulidea.

Flagellobolus Wesener, in: Wesener, Enghoff et Sierwald, 2009. ZooKeys, 19: 41-43. M.

Type-species. - F. pauliana Wesener, in: Wesener, Enghoff et Sierwald, 2009. ZooKeys, 19: 43-44. Madagascar, Périnet; original designation. 1 species.

Pachybolidae, Trigoniulidea.

Gabolus Enghoff, 2008. International Journal of Myriapodology, 2: 148. M.

Type-species. - G. magister Enghoff, 2008. International Journal of Myriapodology, 2: 148-152. Gabon, N'Toum; original designation. 1 species.

Pachybolini, Pachybolinae, Pachybolidae, Trigoniulidea.

Granitobolus Wesener, in: Wesener, Enghoff et Sierwald, 2009. ZooKeys, 19: 66-70. M.

Type-species. - G. endemicus Wesener, in: Wesener, Enghoff et Sierwald, 2009. ZooKeys, 19: 70. Madagascar, Toliara Prov., Ambatotsirongorongo Mountain, Forêt Petit Lavasoa; original designation. 2 species.

Pachybolidae, Trigoniulidea. 54. M

*Hoffmanobolus Shelley, 2001. Myriapodologica, 7:

Type-species. - Hoffmanobolus mexicanus Shelley, 2001. Myriapodologica, 7: 54-56. Mexico, Oaxaca, Cofradia, $12.8 \mathrm{~km}$ ( $8 \mathrm{mi})$ SW San Vicente Lachixo; original designation. 1 species.

Hoffmanobolidae, Spirobolidea.

New family-group name: Hoffmanobolidae Shelley, 2001. Myriapodologica, 7: 54. Family-group component: Hoffmanobolus Shelley, 2001. 
Hylekobolus Wesener, in: Wesener, Enghoff et Sierwald, 2009. ZooKeys, 19: 97-102. M.

Type-species. - H. brachiosauroides Wesener, in: Wesener, Enghoff et Sierwald, 2009. ZooKeys, 19: 102-104. Madagascar, Toliara Prov., Ivorona, west slope Vohimena Mountains; original designation. 11 species.

Spirobolellidae, Spirobolidea.

Hyperbolus Enghoff, 2011. Zootaxa, 2753: 16. M.

Type-species. - H. apicomplexus Enghoff, 2011. Zootaxa, 2753: 16-21. Tanzania, Tanga Reg., East Usambara Mountains, Amani, at Sigi River; original designation. 2 species.

Pachybolini, Pachybolinae, Pachybolidae, Trigoniulidea.

Madabolus Wesener et Enghoff, 2008. Invertebrate Systematics, 22: 43-44. M.

Type-species. - M. maximus Wesener et Enghoff, 2008. Invertebrate Systematics, 22: 44-50. Madagascar, Mahajanga Prov., Parc National de Bemaraha, south bank of Manambolo River, near Tombeau Vazimba, $3.5 \mathrm{~km}$ (2.2 mi) E Bekopaka; original designation. 1 species.

Pachybolini, Pachybolinae, Pachybolidae, Trigoniulidea.

Ostinobolus Wesener, in: Wesener, Enghoff et Sierwald, 2009. ZooKeys, 19: 86-90. M.

Type-species. - O. rufus Wesener, in: Wesener, Enghoff et Sierwald, 2009. ZooKeys, 19: 90-92. Madagascar, Toliara Prov., Andohahela, Malio; original designation. 4 species.

Pachybolidae, Trigoniulidea.

Parabolus Enghoff, 2011. Zootaxa, 2753: 23. M.

Type-species. - Trigoniulus dimorphus Carl, 1909. Revue Suisse de Zoologie, 17: 362-365. Tanzania, Dar-esSalaam; original designation. 1 species.

Pachybolini, Pachybolinae, Pachybolidae, Trigoniulidea.

Pseudocentrobolus Wesener, in: Wesener, Enghoff et Sierwald, 2009. ZooKeys, 19: 57-62. M.

Type-species. - P. aureus Wesener, in: Wesener, Enghoff et Sierwald, 2009. ZooKeys, 19: 62-64. Madagascar, Toliara Prov., Forêt Beroboka, 5.9 km (3.7 mi) SE Ankidranoka; original designation. 2 species.

Pachybolidae, Trigoniulidea.

M.

Quisquellacricus Pérez-Asso, 2004. Solenodon, 4: 17.

Type-species. - Rhinocricus hispaniolus Loomis, 1941 Bulletin of the Museum of Comparative Zoology, 58(3): 39-41. Dominican Republic, La Vega, Jarabacoa; original designation. 3 species.

Rhinocricidae, Rhinocricidea.

*Rhinocricus Karsch, 1881. Zeitschrift für die gesammten Naturwissenschaften, 54: 68. M

Type-species. - Spirobolus parcus Karsch, 1881 Zeitschrift für die gesammten Naturwissenschaften, 54: 68. Puerto Rico; subsequent designation of Pocock [1894: 485]. 200 nominal species [Marek et al., 2003; updated by us].

Rhinocricidae, Rhinocricidea.

New ordinal-group name: Rhinocricidea Pitz et Sierwald, 2010. Cladistics, 26: 512. Ordinal-group component: Rhinocricidae Brölemann, 1913.

Remarks: Pitz \& Sierwald [2010] repeatedly mentioned Rhinocricidea as a new suborder but never provided a for- mal, anatomical, taxonomic account or diagnosis. The Code does not cover ordinal-level taxa, and a sufficient, informal, narrative account, stating anatomical features and that Rhinocricidae is the lone component, is provided in the complete paragraph in the left column on p. 512, so Rhinocricidea is a validly proposed taxon.

Riotintobolus Wesener, in: Wesener, Enghoff et Sierwald, 2009. ZooKeys, 19: 44-51. M.

Type-species. - R. mandenensis Wesener, in: Wesener, Enghoff et Sierwald, 2009. ZooKeys, 19: 51-52. Madagascar, Toliara Prov., Mandena; 4 species.

Pachybolidae, Trigoniulidea.

Sanguinobolus Wesener, in: Wesener, Enghoff et Sierwald, 2009. ZooKeys, 19: 12-14. M.

Type-species. - S. maculosus Wesener, in: Wesener, Enghoff et Sierwald, 2009. ZooKeys, 19: 14-16. Madagascar, Antsiranana Prov., Parc National Montagne d'Ambre, $3.6 \mathrm{~km}(2.3 \mathrm{mi}) \mathrm{SW}$ Joffreville; original designation. 1 species.

Pachybolidae, Trigoniulidea.

Zehntnerobolus Wesener, in: Wesener, Enghoff et Sierwald, 2009. ZooKeys, 19: 38. M.

Type-species. - Spirobolus (Spirostrophus) rubripes DeSaussure et Zehntner, 1897. Myriapodes de Madagascar, in: Grandidier A. (Ed.), Histoire Physique, Naturelle et Politique de Madagascar: 154-159. Madagascar, Toamasina Prov., Forêt d'Andrangoloaka; original designation. 2 species.

Pachybolidae, Trigoniulidea.

\section{SPIROSTREPTIDA}

Agaricogonopus Zhang et Zhang, 1997. Acta Zootaxonomica Sinica, 22(4): 349. M.

Type-species. - A. acrotrifoliolatus Zhang et Zhang, 1997. Acta Zootaxonomica Sinica, 22: 349-351. China, Yunnan Prov., Xishuangbanna (= Xidai); original designation. 1 species.

Remark. Omitted from Shelley et al. [2000].

Rhynchoproctinae, Harpagophoridae, Spirostreptidea.

Alienostreptus Pimvichai, Enghoff et Panha, 2010. Invertebrate Systematics, 24: 58-61. M.

Type-species. - Thyropygus alienus Attems, 1936. Memoirs of the Indian Museum, 11(4): 260-261. Vietnam (S-Annam), Jangbien Prov., Dran; original designation. 1 species.

Rhynchoproctinae, Harpagophoridae, Spirostreptidea.

Apocoptogonus Jeekel, 2006. Myriapod Memoranda, 8: 66-67. M

Type-species. - A. penicillatus Jeekel, 2006. Myriapod Memoranda, 8: 67-71. Australia, New South Wales, Mt. Warning National Park, foot of Mr. Warning; original designation; 2 species.

Cambalidae, Cambalidea.

Aquattuor Frederiksen, 2013. Zootaxa, 3694(1): 64. M.

Type-species. - A. denticulatus Frederiksen, 2013. Zootaxa, 3694(1): 64-65. Tanzania, East Usambara Mountains, Amani; original designation. 1 species

Archepyginae, Odontopygidae, Spirostreptidea. 
Armatostreptus Pimvichai, Enghoff et Panha, 2010. Invertebrate Systematics, 24: 63. M.

Type-species. - Humbertostreptus (?) armatus Demange, 1983. Bulletin du Muséum National d'Histoire Naturelle, série 4, section A, Zoologie, 5(2): 565-567. Thailand, Lampang, Doi Nya Chang near Khun Thal; original designation. 1 species.

Rhynchoproctinae, Harpagophoridae, Spirostreptidea. 32. M.

Cacuminostreptus Mwabvu, 2010. Zootaxa, 2567: 31-

Type-species. - C. mazowensis Mwabvu, 2010. Zootaxa, 2567: 31-32. Zimbabwe, Mazowe Dam; original designation. 4 species.

Spirostreptini, Spirostreptinae, Spirostreptidae, Spirostreptidea.

*Cambalomma Loomis, 1941. Journal of the Washington Academy of Sciences, 31: 189. N.

Type-species. - C. laevis (recte: laeve) Loomis, 1941. Journal of the Washington Academy of Sciences, 31: 189. Haiti; original designation. 1 species.

Cambalomminae, Pseudonannolenidae, Epinannolenidea.

New family-group name: Cambalomminae Mauriès, 1977. Bulletin du Muséum National d'Histoire Naturelle (Sér. 3, no. 431), Zoologie, 301: 248. Family-group component: Cambalomma Loomis, 1941.

Remark: Cambalomminae was omitted from Shelley et al. [2000].

Choristostreptus Hoffman, 2008. Tropical Zoology, 21: 184-185. M.

Type-species. - Triaenostreptus lawrencei Hoffman, 1971. Revue de Zoologie et de Botanique Africaines, 83: 213-218, figs 1-7. Malawi, Blantyre; original designation. 1 species.

Spirostreptidae, Spirostreptidea.

Euryischiogonus Jeekel, 2009. Myriapod Memoranda, 11: 83. M,

Type-species. - E. barbulatus Jeekel, 2009. Myriapod Memoranda, 11: 84-86. Australia, New South Wales, 9 km (5.6 mi) NNE Uralla; original designation. 1 species.

Cambalidae, Cambalidea.

Helicostreptus Hoffman, 2010. International Journal of Myriapodology, 3: 170-171. M.

Type-species. - H. attemsi Hoffman, 2010. International Journal of Myriapodology, 3: 171-174. Sudan, East Equatoria Prov., Gilo, Imatong Mountains; original designation. 1 species.

Spirostreptidae, Spirostreptidea.

Heptischius Pimvichai, Enghoff et Panha, 2010. Invertebrate Systematics, 24: 66-68. M.

Type-species. - H. lactuca Pimvichai, Enghoff et Panha, 2010. Invertebrate Systematics, 24: 68-69. Thailand, Tak, Mae Usu Cave; original designation. 1 species.

Rhynchoproctinae, Harpagophoridae, Spirostreptidea.

Hyloecostreptus Hoffman, 2005. Myriapodologica, 8(9): 83-84. M.

Type-species. - H. shelleyi Hoffman, 2005. Myriapodologica, 8(9): 84-87. Peru, Loreto Dept., farm on Yavari River ca. $81 \mathrm{~km}$ (50.6 mi) upstream of Benjamin Constant, Brazil; original designation. 3 species.
Spirostreptidae, Spirostreptidea.

*Ktenostreptus Attems, 1909. Arkiv för Zoologi, 5(3): 74. M.

Type-species. - K. annulipes Attems, 1909. Arkiv för Zoologi, 5(3): 75. Sri Lanka, Sabaragamuwa Prov., Kuruwita, Point de Galle; monotypy. 10 species.

Ktenostreptini, Harpagophorinae, Harpagophoridae, Spirostreptidea.

New family-group name: Ktenostreptini Jeekel, 2006. Myriapod Memoranda, 9: 35. Family-group components: Ktenostreptus Attems, 1909; Leptostreptus Attems, 1936; Dameptus Attems, 1942; Humbertostreptus Demange, 1969.

Lamelloramus Frederiksen, 2013. Zootaxa, 3694(1): 6061. M.

Type-species. - L. rhombiformis Frederiksen, 2013. Zootaxa, 3694(1): 61-63. Tanzania, East Usambara Mountains, Amani; original designation. 2 species.

Archepyginae, Odontopygidae, Spirostreptidea.

Limnostreptus Hoffman, 2008. Tropical Zoology, 21: 173-177. M.

Type-species. - L. caroli Hoffman, 2008. Tropical Zoology, 21: 177-179. Tanzania, Kigoma Reg. and Dist., Helembe; original designation. 5 species.

Spirostreptidae, Spirostreptidea.

Metaphora Redman, 2003. African Invertebrates, 44(2): 228. F.

Type-species. - Spirostreptus (Nodopyge) spirobolinus Karsch, 1881. Zeitschrift für die gesammten Naturwissenschaften, 54: 28. South Africa, Northern Cape Prov., Hantam; original designation. 1 species.

Harpagophoridae, Spirostreptidea.

Namibostreptus Mwabvu, Hamer, Slotow et Barraclough, 2009. Zootaxa, 2211: 49. M.

Type-species. - Spirostreptus kymatorhabdus Attems, 1914. Zoologica, 25(65/66): 151. Namibia, Walfishbay, Colonial-Gesellschaft; original designation. 1 species.

Spirostreptidae, Spirostreptidea.

Prominulostreptus Pimvichai, Enghoff et Panha, 2010. Invertebrate Systematics, 24: 69. M.

Type-species. - Junceustreptus prominulus Demange, 1961. Mémoires du Muséum National d'Histoire Naturelle, Série A, Zoologie, 24: 239-244. China, Yunnan Prov., Lou Fou Tsouen (Ing Ka Tsoué); original designation. 1 species. Rhynchoproctinae, Harpagophoridae, Spirostreptidea.

*Pseudonannolene Silvestri, 1895. Annali del Museo Civico di Storia Naturale di Genova, Serie 2, 34: 775. F.

Type-species. - P. typica Silvestri, 1895. Annali del Museo Civico di Storia Naturale di Genova, Serie 2, 34: 775. Argentina, Misiones, Candelaria; subsequent designation of Silvestri [1896: 170]. 30 species.

Pseudonannoleninae, Pseudonannolenidae, Epinannolenidea.

New ordinal-group name: Pseudonannolenidea Mauriès, 1983. Bulletin du Muséum National d'Histoire Naturelle, Paris $\{$ Série 4\}, 5(1): 250. Implicitly synonymized with Epinannolenidea Chamberlin, 1922 (misspelled as Epinannolenoidea) by Hoffman [1999: 114]; formally synonymized by Shelley [2003: 196]. Ordinal-group components: Iulomorphidae Verhoeff, 1924; Pseudonannolenidae Silvestri, 
1895; Physiostreptidae Silvestri, 1903; Choctellidae Chamberlin et Hoffman, 1950.

Remarks: New taxon status was not indicated by Mauriès [1983]. Pseudonannolenidea was elevated to full ordinal status by Mauriès [1987: 198] but omitted from Shelley et al. [2000].

Rondostreptus Hoffman, 2011. Zootaxa, 2779: 64. M.

Type-species. - R. kiellandi Hoffman, 2011. Zootaxa, 2779: 64-69. Tanzania, Lindi Dist., Rondo Plateau; original designation. 1 species.

Spirostreptidae, Spirostreptidea.

Sagmatostreptus Hoffman et Enghoff, 2011. Zootaxa, 2903: 21-22. M.

Type-species. - Spirostreptus strongylopygus Attems, 1950. Annalen des Naturhistorischen Museums in Wien, 57 198. Tanzania, West Usambara Mountains (= "W-Usambara"); original designation. 1 species.

Spirostreptidae, Spirostreptidea.

Stenischiogonus Jeekel, 2009. Myriapod Memoranda, 11: 86-87. M.

Type-species. - S. bacillifer Jeekel, 2009. Myriapod Memoranda, 11: 87-90. Australia, New South Wales, $12 \mathrm{~km}$ (7.5 mi) ESE Scone, near Lake Glenbawn; original designation. 1 species.

Cambalidae, Cambalidea.

Taitastreptus VandenSpiegel, 2001. Insect Systematics and Evolution, 32: 475-476. M.

Type-species. - T. flavipes VandenSpiegel, 2002. Insect Systematics and Evolution, 32: 476-480. Kenya, Taita Hills; original designation. 1 species.

Spirostreptidae, Spirostreptidea.

Thiangetastreptus Mwabvu et VandenSpiegel, 2007. Journal of Afrotropical Zoology, 3: 35-36. M.

Type-species. - Spirostreptus montanus Attems, 1909. Wissenschaftliche Ergebnisse der Schwedischen Zoologischen Expedition nach dem Kilimandjaro, dem Meru und dem umgebenden Massaisteppen Deutsch-Ostafrikas 19051906 unter Leitung von Prof. Dr. Yngve Sjöstedt, Stockholm, 3(19): 28-30. Tanzania, Kilimanjaro, Kibonoto; original designation. 2 species.

Spirostreptidae, Spirostreptidea.

Umbraticus Özdikmen, 2009. Munis Entomology and Zoology, 4(1): 182-183. M.

Type-species. - Tomogonus intortus Demange, 1971 Mémoires de l'Institut Fondamental d'Afrique Noire, 86: 208. Sierra Leone, Loma Mountains; direct substitution. 7 species.

Remark: Proposed to replace Tomogonus Demange, 1971, Mémoires de l'Institut Fondamental d'Afrique Noire, 86: 206-208, preoccupied by Tomogonus d'Orbigny, 1904 (Coleoptera), Annali del Museo Civico di Storia Naturale di Genova, 40: 254.

Spirostreptidae, Spirostreptidea

\section{NEMATOPHORA: CHORDEUMATIDA} 42. $\mathrm{F}$

*Altajella Gulička, 1972. Zoologicheskii Zhurnal, 51(1):

Type-species. - A. pallida Gulička, 1972. Zoologicheskii Zhurnal, 51(1): 42. Russia, Siberia, Altai Mountains; original designation. 1 species.
Kirkayakidae, Neoatractosomatoidea, Craspedosomatidea.

New family-group name: Altajellidae Mikhaljova et Golovatch, 2000. Arthropoda Selecta, 9(2): 115-116. Familygroup components: Altajella Gulička, 1972; Teleckophoron Gulièka, 1972; Elongeuma Golovatch, 1982.

Remarks: Because Altajella Gulička, 1972, is preoccupied by Altajella Kulkov, 1962 (Brachiopoda), Özdikmen [2008] proposed the replacement name, Kirkayakus Özdikmen, 2008, thereby also replacing Altajellidae Mikhaljova et Golovatch, 2000, with Kirkayakidae Özdikmen, 2008, nom. nov. 158. F.

Asiatyla Mikhaljova, 2000. Arthropoda Selecta, 8(3):

Type-species. - A. similata Mikhaljova, 2000. Arthropoda Selecta, 8(3): 158. Russia, Siberia, Irkutsk Area, Lake Baikal, environs of Maritui; original designation. 4 species.

Remark: The printed date is 1999 , but the actual publication date was March 2000 (http://kmkjournals.com/journals/AS).

Diplomaragnidae, Diplomaragnoidea, Chordeumatidea.

Banatosoma Ćurčić et Makarov, 2000. Archives of Biological Sciences, Belgrade, 52(3): 175. N.

Type-species. - Bulgarosoma ocellatum Tabacaru, 1967. International Journal of Speleology, 3: 16-19. Romania, Banat, Bohui Cave near Anina; original designation. 1 species.

Anthroleucosomatidae, Anthroleucosomatoidea, Craspedosomatidea.

Belbogosoma Ćurčić et Makarov, 2008. Zootaxa, 1743: 2-4. N.

Type-species. - B. bloweri Ćurčić et Makarov, 2008. Zootaxa, 1743: 4-8. Serbia, Mt. Tupińnica, Gornja Pećina Cave at village of Lenovac; original designation. 2 species.

Anthroleucosomatidae, Anthroleucosomatoidea, Craspedosomatidea.

*Beticosoma Mauriès, 1990. Bulletin de la Société d'Histoire Naturelle de Toulouse, 126: 55, 57. N.

Type-species. - B. longipenis Mauriès, 1990. Bulletin de la Société d'Histoire Naturelle de Toulouse, 126: 55. Spain, Córdoba, Parc Naturel de Hormachuelos; monotypy. 1 species.

Beticosomatidae, Brannerioidea, Craspedocomatidea.

New family-group name: Beticosomatidae Mauriès, 2014. Arthropoda Selecta, 23(1): 37. Family-group component: Beticosoma Mauriès, 1990.

Causeyella Shear, 2003. Zootaxa, 321: 26-27. F.

Type-species. - Scoterpes dendropus Loomis, 1939. Bulletin of the Museum of Comparative Zoology, 86: 181182. USA, Missouri, Stone Co., Marvel Cave; original designation. 3 species.

Trichopetalidae, Cleidogonoidea, Craspedosomatidea.

*(Cyrnosoma) Mauriès, 1969. Annales de Spéléologie, 24(3): 507. N

Type-species. - Not designated, three new species are described in the original publication (see Shelley et al. [2000: 67]). tidea.

Cyrnosomatidae, Neoatractosomatoidea, Craspedosoma-

New family-group name: Cyrnosomatidae Mauriès, 2003. Arthropoda Selecta, 12(1): 16. Family-group component: 
(Cyrnosoma) Mauriès, 1969

Remark: As Cyrnosoma is an unavailable genus-group name and the only component of its family, Cyrnosomatidae is also unavailable.

Dazbogosoma Makarov et Ćurčić, 2012. Archives of Biological Sciences, Belgrade, 64 (2): 801-802. N.

Type-species. - D. naissi Makarov et Ćurčić, 2012. Archives of Biological Sciences, Belgrade, 64 (2): 802-805. Serbia, Cerjanska Propast Cave, village Cerje, near Niš; original designation. 1 species.

Anthroleucosomatidae, Anthroleucosomatoidea, Craspedosomatidea.

*Guizhousoma Mauriès, 2005. Arthropoda Selecta, 14(1): 12. N.

Type-species. - G. latellai Mauriès, 2005. Arthropoda Selecta, 14(1): 12-17. China, Guizhou Prov., Qianzi, Hong Lin (village), Chang-Tu Dong Cave; original designation. 1 species.

Guizhousomatidae, Neoatractosomatoidea, Craspedosomatidea.

New family-group name: Guizhousomatidae Mauriès, 2005. Arthropoda Selecta, 14(1): 12. Family-group component: Guizhousoma Mauriès, 2005.

*Heterochordeuma Pocock, 1893. Annali del Museo Civico di Storia Naturale di Genova, 33: 387. N.

Type-species. - H. doriae Pocock, 1893. Annali del Museo Civico di Storia Naturale di Genova, 33: 387 . Myanmar; monotypy. 4 species.

Heterochordeumatidae, Heterochordeumatoidea, Heterochordeumatidea.

New ordinal-group name: Heterochordeumatidea Shear, 2000. Invertebrate Taxonomy, 14: 373 . Ordinal-group components: Heterochordeumatoidea Pocock, 1894; Conotyloidea Cook, 1896; Diplomaragnoidea Attems, 1907; Pygmaeosomatoidea Carl, 1941.

Remark: In its original proposal, Heterochordeumatidea was labeled a "new name" rather than new suborder.

Infulathrix Shear, 2000. Invertebrate Taxonomy, 14: 368. F. (By analogy, as Metopidiothrix Attems, 1907, is feminine [Jeekel, 1971])

Type-species. - I. siam Shear, 2000. Invertebrate Taxonomy, 14: 158. Thailand, Phuket Island, E of Ton Sui Waterfall; original designation. 1 species.

Heterochordeumatidae, Heterochordeumatoidea, Heterochordeumatidea.

*Kirkayakus Özdikmen, 2008. Munis Entomology and Zoology, 3(1): 343. M.

Type-species. - Altajella pallida Gulička, 1972. Zoologicheskii Zhurnal, 51(1): 42. Russia, Altai Mountains; original designation. 1 species.

Remarks: Proposed as a nom. nov. to replace Altajella Gulička, 1972, preoccupied by Altajella Kulkov, 1962 (Brachiopoda), which also required replacing Altajellidae Mikhaljova et Golovatch, 2000, with Kirkayakidae Özdikmen, 2008. dea.

Kirkayakidae, Neoatractosomatoidea, Craspedosomati-

New family-group name: Kirkayakidae Özdikmen, 2008. Munis Entomology and Zoology, 3(1): 343. Family-group components: Teleckophoron Gulička, 1972; Elongeuma Golovatch, 1982; Tarbagataya Golovatch et Wytwer, 2003;
Kirkayakus Özdikmen, 2008.

Leschius Shear et Leonard, 2004. Zootaxa, 609: 2. M. Type-species. - L. mcallisteri Shear et Leonard, 2004. Zootaxa, 609: 3-6. USA, Washington, Thurston Co., McAllister Springs; original designation. 1 species.

Anthroleucosomatidae, Anthroleucosomatoidea, Craspedosomatidea.

Lipseuma Golovatch, Geoffroy et Mauriès, 2006. Zoosystema, 28(1): 85-86. N.

Type-species. - L. josianae Golovatch, Geoffroy et Mauriès, 2006. Zoosystema, 28(1): 86-90. China, Hubei Prov., Banqiao Co., Cave Chuan Dong Zi; original designation. 2 species.

Kashmireumatidae, Brannerioidea, Craspedosomatidea.

Maritimosoma Mikhaljova, 2000. Arthropoda Selecta, 8(3): 176. N.

Type-species. - Diplomaragna turova Mikhaljova, 1997. Arthropoda Selecta, 6(1/2): 125. Russia, Far East, Maritime Prov., Ussuriysky Nature Reserve; original designation. 5 species.

Remark: The printed date is 1999 , but the actual publication date was March 2000 (http://kmkjournals.com/journals/AS).

Diplomaragnidae, Diplomaragnoidea, Chordeumatidea.

Massarilatzelia Makarov et Rađa, 2011. Zootaxa, 2994: 34-35. F.

Type-species. - M. dugopoljica Makarov et Rađa, 2011. Zootaxa, 2994: 35-38. Croatia, Dalmatia, Balići, Dugopolje village, Kraljeva Peć Cave (= Balića Špilja); original designation. 1 species.

Heterolatzeliidae, Brannerioidea, Craspedosomatidea.

*Microlympia Shear et Leonard, 2003. Zootaxa, 243: 4. F.

Type-species. - Microlympia echina Shear et Leonard, 2003. Zootaxa, 243: 4-10. USA, Washington, Jefferson Co., Alder Creek, ca. 2.4 km (1.5 mi) N Hoh River; original designation. 1 species.

Microlympiidae, Brannerioidea, Craspedosomatidea.

New family-group name: Microlympiidae Shear et Leonard, 2003. Zootaxa, 243: 2, 4. Family-group component: Microlympia Shear et Leonard, 2003.

Nannopetalum Shear, 2003. Zootaxa, 321: 13. N.

Type-species. - Nannopetalum pattersonorum Shear, 2003. Zootaxa, 321: 14-15. USA, Virginia, Prince Edward Co., Hampden-Sydney College campus, Wilson Trail. 3 species.

Trichopetalidae, Cleidogonoidea, Craspedosomatidea.

Orientyla Mikhaljova, 2000. Arthropoda Selecta, 8(3): 170. F.

Type-species. - Diplomaragna bureyinskaya Mikhaljova, 1997. Arthropoda Selecta, 6(1/2): 126. Russia, Far East, Khabarovsk Prov., Verkhne-Bureyinsky Dist.; original designation. 2 species.

Remark: The printed date is 1999, but the actual publication date was March 2000 (http://kmkjournals.com/journals/AS).

Diplomaragnidae, Diplomaragnoidea, Chordeumatidea.

*Osellasoma Mauriès, 1984. Studi Trentini di Scienze Naturali (Acta Biologica), 61: 190. N. 
Type-species. - O. caoduroi Mauriès, 1984. Studi Trentini di Scienze Naturali (Acta Biologica), 61: 191-192. Italy, Trento, Monte Baldo, Altissimo di Nago, Pozzo di Val del Parol; monotypy. 1 species.

Osellasomatini, Trimerophorinae, Neoatractosomatidae, Neoatractosomatoidea, Craspedosomatidea.

New family-group name: Osellasomatini Mauriès, 2003. Arthropoda Selecta, 12(1): 16. Family-group component: Osellasoma Mauriès, 1984.

Pacifiosoma Mikhaljova, 2000. Arthropoda Selecta, 8(3): 169. N.

Type-species. - Diplomaragna cristofer Mikhaljova, 1993. Arthropoda Selecta, 2(2): 19. Russia, Far East, Khabarovsk Prov., Ulchsky Dist.; original designation. 2 species.

Remark: The printed date is 1999, but the actual publication date was March 2000 (http://kmkjournals.com/journals/AS).

Diplomaragnidae, Diplomaragnoidea, Chordeumatidea.

Perunosoma Ćurčić et Makarov, 2007. Canadian Entomologist, 139: 479-480. N.

Type-species. - P. trojanica (recte: trojanicum) Ćurčić et Makarov, 2007. Canadian Entomologist, 139: 480-484. Serbia, near Svrljig, Prekonoška Pećina Cave, village of Prekonoga; original designation. 1 species.

Anthroleucosomatidae, Anthroleucosomatoidea, Craspedosomatidea.

Psichrosoma Mauriès, 2013. Arthropoda Selecta, 22(2): 105. N.

Type-species. - Psychrosoma tarraconense Mauriès, 1971. Bulletin de la Société d'Histoire Naturelle de Toulouse, 106: 417, 419. Spain, Tarragona, two caves; original designation of Psychrosoma Mauriès, 1971. 3 species.

Remarks: Psichrosoma, nom. nov. was proposed to replace Psychrosoma Mauriès, 1971, which is preoccupied by Psychrosoma Tapparone-Canefri, 1876 (Mollusca). Additionally, the name of the type-species was originally misspelled as "tarraconensis" but was corrected to "tarraconense" by Mauriès [2013]. tidea.

Vandeleumatidae, Craspedosomatoidea, Craspedosoma-

Pyrgeuma Shear, 2013. Zootaxa, 3560: 80 . N.

Type-species. - P. pyrgodesmoides Shear, 2012. Zootaxa, 3560: 80-84. Malaysia, Western Malaysia, Pahang, Cameron Highlands, Mt. Perdah; original designation. 1 species.

Heterochordeumatidae, Heterochordeumatoidea, Heterochordeumatidea.

Rhodoposoma Ćurčić et Makarov, 2000. Archives of Biological Sciences, Belgrade, 52(3): 175. N.

Type-species. - Bulgarosoma rhodopinum Strasser, 1966. Annales Zoologici, Warszawa, 23: 345-348. Bulgaria, Central Rhodopes near Mostovo, Erkjuprija Cave. 1 species.

Anthroleucosomatidae, Anthroleucosomatoidea, Craspedosomatidea.

Serbosoma Ćurčić et Makarov, 2000. Archives of Biological Sciences, Belgrade, 52(3): 174-175. N.

Type-species. - Bulgarosoma crucis Strasser, 1960. Fragmenta Entomologica, Roma, 3(6): 118-123. Serbia, near Ćuprija, Ravanicka Pécina Cave. 5 species.
Anthroleucosomatidae, Anthroleucosomatoidea, Craspedosomatidea.

Shearia Mikhaljova, 2000. Arthropoda Selecta, 8(3): 162. F.

Type-species. - S. khakassica Mikhaljova, 2000. Arthropoda Selecta, 8(3): 163. Russia, Krasnoyarsk Prov., Khakassian Republic, Shira Dist., ca. 1 km (0.6 mi) S Kommunar; original designation. 7 species.

Remark: The printed publication date is 1999 , but the actual publication date was March 2000 (http:// kmkjournals.com/journals/AS). tidea.

Diplomaragnidae, Diplomaragnoidea, Heterochordeuma80. N.

Sireuma Reboleira et Enghoff, 2014. Zootaxa, 3785(1):

Type-species. - S. nobile Reboleira et Enghoff, 2014. Zootaxa, 3785(1): 80-84. Portugal, Alentejo Prov., Alandroal, Algar de Santo António; original designation. 1 species.

Opisthocheiridae, Cleidogonoidea, Craspedosomatidea.

Svarogosoma Makarov, 2003. Periodicum Biologorum, 108(4): 466. N.

Type-species. - S. bozidarcurcici Makarov, 2003. Periodicum Biologorum, 108(4): 466-467. Serbia, Mt. Suva Planina, Mali Trem, Savina Propast Cave; original designation. 1 species.

Anthroleucosomatidae, Anthroleucosomatoidea, Craspedosomatidea.

Taiwaneuma Mikhaljova, Golovatch et Chang, 2011. Zootaxa, 2980: 50. N.

Type-species. - T. crinitum Mikhaljova, Golovatch et Chang, 2011. Zootaxa, 2980: 50-55. Taiwan, Ilan Co., Datong Township, near Lakes Jialuohu; original designation. 2 species.

Niponiosomatidae, Brannerioidea, Craspedosomatidea.

Tarbagataya Golovatch et Wytwer, 2003. Annales Zoologici, 53(3): 580. F.

Type-species. - T. splendida Golovatch et Wytwer, 2000. Annales Zoologici, 53(3): 580-583. Kazakhstan, Makanchi Dist., Tarbagatay Mountains, Sholakterek River Valley nr. Kirovka (= Karatuma); original designation. 2 species.

Kirkayakidae, Neotractosomatoidea, Craspedosomatidea.

\section{CALLIPODIDA}

Angulifemur Zhang, 1997. Diplopoda from Yunnan Caves, 3. Thesis Compilation of Tianjin Natural History Museum, 14: 1-2. N. (By analogy, as "femur" in Latin is neuter).

Type-species. - A. tridigitis Zhang, 1997. Diplopoda from Yunnan Caves, 3. Thesis Compilation of Tianjin Natural History Museum, 14: 2. China, Yunnan Prov., Mengxi Co., Yang fen Cave; original designation. 2 species.

Remark: Omitted from Shelley et al. [2000].

Paracortinidae, Schizopetalidae.

*Aspidiophon Shelley, 2000. Myriapodologica, 6: 85. N. Type-species. - A. divisum Shelley, 2000. Myriapodologica, 6: 85. Mexico, Sinaloa, $3.2 \mathrm{~km}(2 \mathrm{mi}) \mathrm{N}$ Piaxtla; original designation. 1 species.

Aspidiophonini, Tynommatinae, Tynommatidae, Schizopetalidea. 
New family-group name: Aspidiophonini Shelley, 2000. Myriapodologica, 6: 83. Family-group component: Aspidiophon Shelley, 2000.

Mexicopetalum Stoev et Shelley, 2009. Virginia Museum of Natural History Special Publication 16: 161. N.

Type-species. - M. hoffmani Stoev et Shelley, 2009. Virginia Museum of Natural History Special Publication 16: 161-163. Mexico, Sonora, near Alamos; original designation. 1 species.

Tynommatidae, Schizopetalidea.

*Schizopetalum Verhoeff, 1900. Zoologische Jahrbücher, Abtheilung für Systematik, Geographie und Biologie der Thiere, 13: 52 . N.

Type-species. - Lysiopetalum koelbeli Verhoeff, 1895. Zoologischer Anzeiger, 18: 207. "Yugoslavia"; subsequent designation of Verhoeff [1909: 213]. 1 species.

Schizopetalidae, Schizopetalidea.

New ordinal-group name: Schizopetalidea Hoffman, 1973, Mitteilungen aus dem Hamburgischen Zoologischen Museum und Institut, 69: 83. Ordinal-group component: Schizopetalidae Verhoeff, 1909.

Remark: Omitted from Shelley et al. [2000].

Scotopetalum Shear, 2000. Myriapodologica, 6: 96. N. Type-species. - S. warreni Shear, 2000. Myriapodologica, 6(11): 96-97. Vietnam, NW of Hanoi, unnamed cave at Hong Mat; original designation. 1 species.

Remark: Scotopetalum Shear, 2000, is now considered a junior synonym of Paracortina Wang et Zhang, 1993 [Stoev, Geoffroy, 2004].

Schizopetalidae, Schizopetalidea.

*Sinocallipus Zhang, 1993. Proceedings of the XI International Congress of Speleology, Beijing, China, 129. M.

Type-species. - S. simplipodicus Zhang, 1993. Proceedings of the XI Congress of Speleology, Beijing, China, 129. China, Yunnan Prov., Hekou Yaozu, Xiao Cave; original designation. 6 species.

Sinocallipodidae, Sinocallipodidea.

New ordinal-group name: Sinocallipodidea Shear, 2000. Myriapodologica, 6: 99. Ordinal-group component: Sinocallipodidae Zhang, 1993.

\section{STEMMIULIDA}

Eostemmiulus Mauriès, Golovatch et Geoffroy, 2010. Arthropoda Selecta, 19(2): 77. M.

Type-species. - E. caecus Mauriès, Golovatch et Geoffroy, 2010. Arthropoda Selecta, 19(2): 77-79. Vietnam, Kien Chiang Prov., Kien Luong, Hon Chong, Nui Hills, Bai Voi, hospital cave Mo So; original designation. 1 species.

Stemmiulidae.

\section{NEMATOPHORA: SIPHONIULIDA.}

No new genus- or family-group taxa were proposed in Siphoniulida from 2000-2014.

\section{POLYDESMIDA}

$\mathrm{M}$.

Acerhacus Hoffman, 2006. Myriapodologica, 8(10): 98.

Type-species. - A. oxylomus Hoffman, 2006. Myriapodologica, 8(10): 98-100. Papua New Guinea, Morobe
Dist., ca. $70 \mathrm{~km}(43.7 \mathrm{mi}) \mathrm{W}$ Wau, between Aseki and Haintiba; original designation. 2 species.

Psaphodesminae, Platyrhacidae, Platyrhacoidea, Leptodesmidea.

Adisiella Golovatch, 2000. Amazoniana, 15(3/4): 222. F.

Type-species. - A. circularis Golovatch, 2000. Amazoniana, 15(3/4): 223. Brazil, Amazônas, environs of Manaus, Reserva Florestal A. Ducke; original designation. 3 species.

Remark: The printed date is December 1999, but the actual publication date was April 2000 (Adis, in litt.).

Pyrgodesmidae, Pyrgodesmoidea, Polydesmidea.

Aenictogomphus Hoffman, 2005. Monograph of the Gomphodesmidae, a family of African polydesmoid millipeds. Verlag des Naturhistorischen Museums Wien, 505. M.

Type-species. - Mychodesmus rectus Demange, 1966. Bulletin du Muséum National d'Histoire Naturelle, 62(3): 747. Togo, Klouto; original designation. 1 species.

Gomphodesminae, Gomphodesmidae, Xystodesmoidea, Leptodesmidea.

Aethalosoma Jeekel, 2006. Myriapod Memoranda, 8: 84-85. N.

Type-species. - A. solum Jeekel, 2006. Myriapod Memoranda, 8: 85-89. Australia, South Australia, Ben Lomond National Park, 35 km (21.9 mi) NE Evandale; original designation. 1 species.

Antichiropodini, Australiosomatinae, Paradoxosomatidae, Strongylosomatidea.

Agrophogonus Hoffman, 2005. Monograph of the Gomphodesmidae, a family of African polydesmoid millipeds. Verlag des Naturhistorischen Museums Wien, 474. M.

Type-species. - A. mangalisa Hoffman, 2005. Monograph of the Gomphodesmidae, a family of African polydesmoid millipeds. Verlag des Naturhistorischen Museums Wien, 483. Tanzania, Dodoma Reg., Mpwapwa Dist., 48 km (30 mi) W Kilosa, Rubeho Mountains, Mangalisa Peak; original designation. 5 species.

Gomphodesminae, Gomphodesmidae, Xystodesmoidea, Leptodesmidea.

Amnestorhacus Hoffman et Martínez-Torres, 2012. Annali del Museo Civico di Storia Naturale "G. Doria," 104: 133-134. M.

Type-species. - Arcidesmus ologona Silvestri, 1898. Anales del Museo Nacional de Buenos Aires, 6: 67-68. Colombia, Meta Dept., Villavicencio; original designation. 1 species.

Platyrhacidae, Platyrhacoidea, Leptodesmidea.

Aporodesmella Golovatch, Geoffroy et VandenSpiegel, 2014. ZooKeys, 414: 36. F.

Type-species. - A. securiformis Golovatch, Geoffroy et VandenSpiegel, 2013. ZooKeys, 414: 37-40. Vietnam, Kien Giang Prov., Kien Luong, Hon Chong, Nui Bai Voi; original designation. 3 species.

Trichopolydesmidae, Trichopolydesmoidea, Polydesmidea.

Appalachioria Marek et Bond, 2006. Molecular Phylogenetics and Evolution, 41: 725. F.

Type-species. - Brachoria falcifera Keeton, 1959. Proceedings of the United States National Museum, 109: 27. 
USA, Virginia, Buchanan Co., Grimleysville; original designation. 5 species.

Apheloriini, Xystodesminae, Xystodesmidae, Xystodesmoidea, Leptodesmidea.

Arbelorhacus Hoffman, 2001. Revue suisse de Zoologie, 108(2): 435. M.

Type-species. - A. magirus Hoffman, 2001. Revue suisse de Zoologie, 108(2): 435-437. Malaysia, Eastern Malaysia, Sarawak; original designation. 2 species.

Platyrhacinae, Platyrhacidae, Platyrhacoidea, Leptodesmidea.

Asticopyrgodesmus Mesibov, 2012. ZooKeys, 217: 6566. M.

Type-species. - A. maiala Mesibov, 2012. ZooKeys, 217: 66-69. Australia, Queensland, D'Aguilar National Park, Maiala Section; 2 species.

Pyrgodesmidae, Pyrgodesmoidea, Polydesmidea

Atalopharetra Mesibov, 2005. Zootaxa, 1034: 22-23. F. Type-species. - A. johnsi Mesibov, 2005. Zootaxa, 1034: 23-28. Australia, Tasmania, Tarraleah; original designation. 4 species.

Family not assigned, Dalodesmidea.

Atlantodesmus Hoffman, 2000. Myriapodologica, 6: 102-103. M,

Type-species. - Leptodesmus (Odontopeltis) eimeri Attems, 1898. Denkschriften der Kaiserlichen Akademie der Wissenschaften in Wien, Mathematisch-naturwissenschaftliche Classe, 67: 400. Brazil, Santa Catarina, Blumenau; original designation. 5 species.

Chelodesminae, Chelodesmidae, Chelodesmoidea, Leptodesmidea.

Atrophotergum Mesibov, 2004. Zootaxa, 480: 2-7. N.

Type-species. - Atrophotergum silvaticum Mesibov, 2004. Zootaxa, 480: 8-9. Australia, Tasmania, Winter Brook; original designation. 6 species.

Dalodesmidae, Dalodesmidea.

Auliscodesmus Hoffman, 2005. Monograph of the Gomphodesmidae, a family of African polydesmoid millipeds. Verlag des Naturhistorischen Museums Wien, 201. M

Type-species. - A. jacquelinae Hoffman, 2005. Monograph of the Gomphodesmidae, a family of African polydesmoid millipeds. Verlag des Naturhistorischen Museums Wien, 201. Zimbabwe, Chiradzi; original designation. 1 species.

Aulodesmini, Gomphodesminae, Gomphodesmidae, Xystodesmoidea, Leptodesmidea.

*Aulodesmus Cook, 1896. Proceedings of the United States National Museum, 18: 83. M

Type-species. - Polydesmus mossambicus Peters, 1855. Bericht über die Bekanntmachung geeigneten Verhandlungen der Königlichen Preussischen Akademie der Wissenschaften zu Berlin, 1855: 81. Mozambique, Nampula Prov., Island of Mozambique; original designation. 8 species.

Aulodesmini, Gomphodesminae, Gomphodesmidae, Xystodesmoidea, Leptodesmidea.

New family-group name: Aulodesmini Hoffman, 2005. Monograph of the Gomphodesmidae, a family of African polydesmoid millipeds. Verlag des Naturhistorischen Muse- ums Wien, 174. Family-group components: Aulodesmus Cook, 1896; Tymbodesmus Cook, 1897; Emphsemastix Hoffman, 1966; Auliscodesmus Hoffman, 2005; Streptelopus Hoffman, 2005; Mitumbagomphus Hoffman, 2005; Masaigomphus Hoffman, 2005.

Baianassa Hoffman, 2012. Papéis Avulsos de Zoologia, 52(8): 107. F.

Type-species. - Leptodesmus carminatus Brölemann, 1903. Annales de la Société Entomologique de France, 72: 670. Brazil, Bahia, Condeúba (= Santo Antonio da Barra); original designation. 1 species.

Chelodesminae, Chelodesmidae, Chelodesmoidea, Leptodesmidea

Balkanodesmus Antić et Reip, in: Antić, Reip, Dražina, Rađa et Makarov, 2014. Zootaxa, 3884(2): 102. M.

Type-species. - B. biokovensis Antić et Reip, in: Antić, Reip, Dražina, Rađa et Makarov, 2014. Zootaxa, 3884(2): 102-106. Croatia, Mt. Biokovo, Saint Jure, Amfora Pit; original designation. 1 species.

Trichopolydesmidae, Trichopolydesmoidea, Polydesmidea.

Belousoviella Golovatch, 2012. Arthropoda Selecta, 21(1): 8. F.

Type-species. - B. kabaki Golovatch, 2012. Arthropoda Selecta, 21(1): 8. China, Sichuan Prov., SW of Mianning, right tributary of Yalongjiang River Canyon; original designation. 1 species.

Sulciferini, Paradoxosomatinae, Paradoxosomatidae, Strongylosomatidea.

Beronodesmus Golovatch, 2014. Arthropoda Selecta, 23(3): 248. M.

Type-species. - B. pallidus Golovatch, 2014. Arthropoda Selecta, 23(3): 248-250. Nepal, Langtang Valley, Kyangjin, Gompa Forest; original designation. 1 species.

Chamberliniini, Paradoxosomatinae, Paradoxosomatidae, Strongylosomatidea.

Betarhacus Hoffman, 2006. Myriapodologica, 8(10): 104-106. M.

Type-species. - Platyrrhacus fallens Chamberlin, 1920. Bulletin of the Museum of Comparative Zoology, 54: 142. Republic of the Solomon Islands, Fulakora; original designation. 1 species.

Psaphodesminae, Platyrhacidae, Platyrhacoidea, Leptodesmidea.

Binarcifer Mesibov, 2013. Memoirs of the Queensland Museum - Nature, 58: 11. M.

Type-species. - B. superbus Mesibov, 2013. Memoirs of the Queensland Museum - Nature, 58: 11-13. Australia, Queensland, Mt. Superbus; original designation. 1 species.

Australiosomatini, Australiosomatinae, Paradoxosomatidae, Strongylosomatidea.

Brachytelopus Hoffman, 2005. Monograph of the Gomphodesmidae, a family of African polydesmoid millipeds. Verlag des Naturhistorischen Museums Wien, 442. M.

Type-species. - Gomphodesmus parvulus Attems, 1929. Revue de Zoologie et de Botanique Africaines, 17: 364. Democratic Republic of the Congo, Sud-Kivu Dist., Beni Terr., Kasindi; original designation. 1 species.

Gomphodesminae, Gomphodesmidae, Xystodesmoidea, Leptodesmidea. 
Bromodesmus Mesibov, 2004. Zootaxa, 558: 4-8. M. Type-species. - B. catrionae Mesibov, 2004. Zootaxa, 558: 8-9. Australia, Tasmania, Weavers Creek; original designation. 4 species.

Dalodesmidae, Dalodesmidea.

Carinorthomorpha Golovatch, 2000. Arthropoda Selecta, 8(4): 215. F.

Type-species. - C. minuta Golovatch, 2000. Arthropoda Selecta, 8(4): 215-218. Thailand, Chieng Mai Prov., Doi Inthanon; original designation. 1 species.

Remark: The printed publication is dated 1999, but the actual date was June 2000 (http://kmkjournals.com/journals/ AS).

Orthomorphini, Paradoxosomatinae, Paradoxosomatidae, Strongylosomatidea.

Cemsunguria Özdikmen, 2007. Munis Entomology and Zoology, 2(2): 433-434. F.

Type-species. - Szechuanella tenebra Hoffman, 1961 Annals and Magazine of Natural History, 3: 533-535. China, Szechuan Prov., Wushan; original designation. 2 species.

Remarks: Although Özdikmen [2007] proposed Cemsunguria, nom. nov., to replace Szechuanella Hoffman, 1960, preoccupied by Szechuanella Zhang et Fan, 1960 (Trilobita), he did not realize that Hoffman's name had previously been replaced with Sellanucheza Enghoff, Golovatch et Nguyen, 2004. Consequently, Cemsunguria Özdikmen, 2007, is a junior objective synonym of Sellanucheza Enghoff, Golovatch et Nguyen, 2004.

Tonkinosomatini, Paradoxosomatinae, Paradoxosomatidae, Strongylosomatidea.

Centrocladus Jeekel, 2006. Myriapod Memoranda, 9: 103-106. M.

Type-species. - Isocladosoma (Centrocladus) dissimile Jeekel, 2006. Myriapod Memoranda, 9: 103-106. Australia, New South Wales, Wauchope, Doyles S. F., Fenwicks Rd.; monotypy. 1 species.

Remark: Proposed as a subgenus of Isocladosoma Jeekel, 1984; the descriptions of the new subgenus and species are the same.

Australiosomatini, Australiosomatinae, Paradoxosomatidae, Strongylosomatidea.

*Chelodesmus Cook, in: Cook et Collins, 1895. Annals of the New York Academy of Sciences, 9: 4. M.

Type-species. - C. marxi Cook, 1895, in: Cook et Collins, 1895 [Cook, Collins, 1895: 4]; locality unknown; original designation. $\sim 17$ species in 1980.

Remark: Chelodesmus is now considered a synonym of Eurydesmus DeSaussure, 1860 [Hoffman, 1980].

Chelodesmini, Chelodesminae, Chelodesmidae, Chelodesmoidea, Leptodesmidea.

New ordinal-group name: Chelodesmidea Hoffman, 1967. Revue de Zoologie et de Botanique Africaines, 76: 209. Ordinal-group components: Chelodesmoidea Cook, 1895; Platyrhacoidea Pocock, 1895; Rhachodesmoidea Carl, 1903; Sphaeriodesmoidea Humbert et DeSaussure, 1869; Xystodesmoidea Cook, 1895.

Remark: Omitted from Shelley et al. [2000] and now considered a synonym of Leptodesmidea Brölemann. 1916 [Shelley, 2003].
Chinomorpha Golovatch, 2011. Arthropoda Selecta, 20(4): 259-260. F.

Type-species. - C. montana Golovatch, 2011. Arthropoda Selecta, 20(4): 260. China, Yunnan Prov., S of Nixi; original designation. 1 species.

Remark: Chinomorpha Golovatch, 2011, is now considered a synonym of Sigipinius Hoffman, 1961 [Golovatch, 2013].

Sulciferini, Paradoxosomatinae, Paradoxosomatidae, Strongylosomatidea.

Clastrorhacus Hoffman, 2006. Myriapodologica, 8(10): 102. M.

Type-species. - Platyrhacus acmophorus Chamberlin, 1945. American Museum of Natural History Novitates, 1282: 16. Indonesia, West Papua (= Irian Jaya), Doormanpad; original designation. 1 species.

Psaphodesminae, Platyrhacidae, Platyrhacoidea, Leptodesmidea.

Clastrotylus Hoffman, 2005. Monograph of the Gomphodesmidae, a family of African polydesmoid millipeds. Verlag des Naturhistorischen Museums Wien, 299. M.

Type-species. - Eurydesmus luridus Karsch, 1881. Archiv für Naturgeschichte, 47: 43. Kenya, Mombasa; original designation. 2 species.

Astrodesmini, Gomphodesminae, Gomphodesmidae, Xystodesmoidea, Leptodesmidea.

Corymborhacus Hoffman, 2006. Myriapodologica, 8(10): 94-95. M.

Type-species. - C. manus Hoffman, 2006. Myriapodologica, 8(10): 96-97. Papua New Guinea, Admiralty Islands, Manus Island; original designation. 1 species.

Psaphodesminae, Platyrhacidae, Platyrhacoidea, Leptodesmidea.

Creagronopus Hoffman, 2001. Revue suisse de Zoologie, 108(2): 416. M.

Type-species. - Hoplurorhachis hosei Pocock, 1897. Annals and Magazine of Natural History, (6), 20: 438. Borneo, Malaysia, East Malaysia, Sarawak; original designation. 2 species.

Hoplurorhachini, Platyrhacinae, Platyrhacidae, Platyrhacoidea, Leptodesmidea.

Cryptosolenomeris Hoffman, 2009. Papéis Avulsos de Zoologia, 49(42): 558. F. (As gender is not provided in the original description, we accept Jeekel's [1971: 9-10] resolution for names ending in "meris" in the order Glomerida and consider it feminine).

Type-species. - C. macrogon Hoffman, 2009. Papéis Avulsos de Zoologia, 49(42): 558-561. Brazil, Espírito Santo, Linhares; original designation. 1 species.

Chelodesminae, Chelodesmidae, Chelodesmoidea, Leptodesmidea.

Curimaguana Hoffman, 2007. Myriapodologica, 9(1): 1. F.

Type-species. - Curimagua granulata Hoffman, 1982. Journal of Natural History, 16: 646-648. Venezuela, Falcon Edo., Serrania de San Luis, Valle de Curimagua, Cueva de Camburales; direct substitution. 1 species.

Remarks: Proposed to replace Curimagua Hoffman, 1982, Journal of Natural History, 16: 645, preoccupied by Curimagua Forster et Platnick, 1976 (Araneae), American 
Museum Novitates, 2619: 24. Özdikmen [2009] subsequently proposed Vigilia, nom.nov., to replace Curimagua Hoffman, 1982, but this name is actually a junior objective synonym of Curimaguana Hoffman, 2007.

Batodesmini, Chelodesminae, Chelodesmidae, Chelodesmoidea, Leptodesmidea.

Dasystigma Mesibov, 2003. Memoirs of Museum Victoria, 60(2): 198-199, 202. N.

Type-species. - Lissodesmus margaretae Jeekel, 1984. Papers and Proceedings of the Royal Society of Tasmania, 118: 99-101. Australia, Tasmania, Lake Augusta; original designation. 4 species.

Dalodesmidae, Dalodesmidea.

Deharvengius Golovatch, Geoffroy et VandenSpiegel, 2013. ZooKeys, 414: 44-45. M.

Type-species. - D. bedosae Golovatch, Geoffroy et VandenSpiegel, 2013. ZooKeys, 414: 46-48. Vietnam, Kien Giang Prov., Kien Luong, Hon Chong, Nui Bai Voi; original designation. 1 species.

Trichopolydesmidae, Polydesmidea.

Delirus Özdikmen, 2009. Munis Entomology and Zoology, 4(1): 181. M.

Type-species. - Cylindromus uniporus Loomis, 1977. Florida Entomologist, 60(1): 22. Puerto Rico, Cuevo los Choros, $15 \mathrm{~km}$ (9.4 mi) S Arecibo; direct substitution. 1 species.

Remarks: Proposed to replace Cylindromus Loomis, 1977, Florida Entomologist, 60(1): 22, preoccupied by $C y$ lindromus Aurivillius, 1891 (Coleoptera), Nouvelles Archives du Muséum National d'Histoire Naturelle, Paris, 7(3): 213 Because Cylindromus Loomis, 1977, has been synonymized under Ricodesmus Chamberlin, 1922 [Pérez-Asso, 2002], Delirus Özdikmen, 2009, automatically falls in the same synonymy.

Chelodesminae, Chelodesmidae, Chelodesmoidea, Leptodesmidea

Dendrodesmus Pérez-Asso, 2003. Solenodon, 3: 57. M. Type-species. - D. yuma Pérez-Asso, 2003. Solenodon, 3: 58-59. Dominican Republic, Prov. La Altagracia, Parque Nacional del Este near Boca de Yuma, path to Bienvo Cave; original designation. 1 species.

Chelodesminae, Chelodesmidae, Chelodesmoidea, Leptodesmidea.

Desmoxytoides Mesibov, 2006. Zootaxa, 1354: 32-33. M Type-species. - D. hasenpuschorum Mesibov, 2006. Zootaxa, 1354: 33-37. Australia, Queensland, Kirrama Range, Mt. Hosie; original designation. 1 species.

Australiosomatinae, Paradoxosomatinae, Paradoxosomatidae, Strongylosomatidea.

Remark: Although the tribal position is not indicated in the original description, Desmoxytoides has since been allocated to Australiosomatinae [Golovatch et al., 2012].

*Diallagmogon Hoffman, 2005. Monograph of the Gomphodesmidae, a family of African polydesmoid millipeds. Verlag des Naturhistorischen Museums Wien, 374. M.

Type-species. - Gomphodesmus testaceus Attems, 1909. Zoologische Jahrbücher, Abteilung für Systematik, Geographie und Biologie der Tiere. 27: 410. Ethiopia, Kefa Prov., probably in Omo drainage between Gardula and headwaters of the Gilo River (South Kaffa: Dereta Mountains and Buka Wari (Kaffa)); original designation. 1 species.

Diallagmogonini, Gomphodesminae, Gomphodesmidae, Leptodesmidea.
New family-group name: Diallagmogonini Hoffman, 2005. Monograph of the Gomphodesmidae, a family of African polydesmoid millipeds. Verlag des Naturhistorischen Museums Wien, 372. Family-group component: Diallagmogon Hoffman, 2005.

*Dibolostethus Hoffman, 2009. International Journal of Myriapodology, 2: 151. M.

Type-species. - D. sicarius Hoffman, 2009. International Journal of Myriapodology, 2: 151-154. Ecuador, Pichinchi Prov., 56 km (35 mi) N Quevedo, Rio Palenque Science Center; original designation; 1 species.

Dibolostethini, Chelodesminae, Chelodesmidae, Chelodesmoidea, Leptodesmidea.

New family-group name: Dibolostethini Hoffman, 2009. International Journal of Myriapodology, 2: 150-151. Family-group component: Dibolostethus Hoffman, 2009.

*Diodontodesmus Pocock, 1897. Annals and Magazine of Natural History (6), 20: 443. M.

Type-species. - D. woodfordi Pocock, 1897. Annals and Magazine of Natural History (6), 20: 443. Republic of the Solomon Islands, Buka Island; original designation. 3 species.

Diodontodesminae, Platyrhacidae, Platyrhacoidea, Leptodesmidea.

New family-group name: Diodontodesmini Jeekel, 2007. Myriapod Memoranda, 10: 84, 93, elevated to subfamilial status by Shelley \& Martínez-Torres [2013]. Family-group components: Diodontodesmus Pocock, 1897; Paradesmorhachis Pocock, 1897.

Dorcadogonus Jeekel, 2000. Entomologica Scandinavica, 30: 476. M.

Type-species. - D. modestus Jeekel, 2000. Entomologica Scandinavica, 30: 476-478. Republic of the Solomon Islands, Rennell Island, Niupani; original designation. 1 species.

Aschistodesmini, Australiosomatinae, Paradoxosomatidae, Strongylosomatidea.

Dysmicodesmus Mesibov, 2010. Zootaxa, 2571: 58. M.

Type-species. - D. jeekeli Mesibov, 2010. Zootaxa, 2571: 58-61. Australia, Tasmania, Blackwater Road; original designation. 1 species.

Family not assigned, Dalodesmidea.

Ebanodesmus Pérez-Asso, 2005. Solenodon, 5: 60-61. M Type-species. - E. victori Pérez-Asso, 2005. Solenodon, 5: 62. Dominican Republic, La Vega Prov., Ebano Verde Scientific Reserve; monotypy. 1 species.

Chelodesminae, Chelodesmidae, Chelodesmoidea, Leptodesmidea. 10. F.

Ectopotremia Hoffman, 2007. Myriapodologica, 9(3):

Type-species. - E. pierrardi Hoffman, 2007. Myriapodologica, 9(3): 11-12. Mali, Sikasso State, 95 km (59.4 mi) SE Ségou; original designation. 1 species.

Prepodesminae, Chelodesmidae, Chelodesmoidea, Leptodesmidea.

*Elaphogonus Attems, 1909. Wissenschaftliche Ergebnisse der Schwedischen Zoologischen Expedition nach dem Kilimandjaro, dem Meru und dem umgebenden Massaisteppen Deutsch-Ostafrikas 1905-1906 unter Leitung von Prof. Dr. Yngve Sjöstedt, Stockholm, 3(19): 12. M. 
Type-species. - E. carneus Attems, 1909. Wissenschaftliche Ergebnisse der Schwedischen Zoologischen Expedition nach dem Kilimandjaro, dem Meru und dem umgebenden Massaisteppen Deutsch-Ostafrikas 1905-1906 unter Leitung von Prof. Dr. Yngve Sjöstedt, Stockholm, 3(19): 12. Tanzania, "Meru-Niederung, an der westlichen Seite des Berges" (= Meru Lowland, W side of mountain); original designation. 3 species.

Elaphogonini, Marptodesminae, Gomphodesmidae, Xystodesmoidea, Leptodesmidea.

New family-group name: Elaphogonini Hoffman, 2005. Monograph of the Gomphodesmidae, a family of African polydesmoid millipeds. Verlag des Naturhistorischen Museums Wien, 68. Family-group components: Elaphogonus Attems, 1909; Endecaporus Brolemann, 1920; Uluguria Hoffman, 1964; Giryama Hoffman, 2005; Kilimagomphus Hoffman, 2005.

Emplectomastix Hoffman, 2005. Monograph of the Gomphodesmidae, a family of African polydesmoid millipeds. Verlag des Naturhistorischen Museums Wien, 466. F. (By analogy, as "mastix" in Greek is feminine).

Type-species. - E. mirabilis Hoffman, 2005. Monograph of the Gomphodesmidae, a family of African polydesmoid millipeds. Verlag des Naturhistorischen Museums Wien, 468. Tanzania, Utete Dist., Matumbi Uplands, Kiwengoma Forest Reserve; original designation. 1 species.

Gomphodesminae, Gomphodesmidae, Xystodesmoidea, Leptodesmidea.

*Erythrhacus Hoffman, 1962. Annals and Magazine of Natural History, 13: 23. M.

Type-species. - Pachyurus erythrokrepis Attems, 1897. Abhandlungen der Senckenbergischen Naturforschenden Geselleschaft, 23: 489. Indonesia, Sulawesi, NE of Minahassa; original designation. 10 species.

Erythrhacinae, Platyrhacidae, Platyrhacoidea, Leptodesmidea.

New family-group name: Erythrhacini Jeekel, 2007. Myriapod Memoranda, 10: 81, 92, elevated to subfamilial status by Shelley \& Martínez-Torres [2013]. Family-group components: Derodesmus Cook, 1896; Ilodesmus Cook, 1896; Erythrhacus Hoffman, 1962; Mastigorhacus Jeekel, 2007.

*Erythranassa Hoffman et Howell, 1987. Revue de Zoologie Africaine, 100: 473. F.

Type-species. - E. saucra Hoffman et Howell, 1987. Revue de Zoologie Africaine, 100: 474. Tanzania, Coast Region, Kisarawe, Pugu Forest Reserve; original designation. 1 species.

Erythranassini, Gomphodesminae, Gomphodesmidae, Xystodesmoidea, Leptodesmidea.

New family-group name: Erythranassini Hoffman, 2005. Monograph of the Gomphodesmidae, a family of African polydesmoid millipeds. Verlag des Naturhistorischen Museums Wien, 279. Family-group component: Erythranassa Hoffman et Howell, 1987.

Eungellosoma Mesibov, 2013. Memoirs of the Queensland Museum - Nature, 58: 7-8. N.

Type-species. - E. leichhardti Mesibov, 2013. Memoirs of the Queensland Museum - Nature, 58: 8-11. Australia, Queensland, Eungella National Park, Dalrymple Road; original designation. 1 species.

Australiosomatini, Australiosomatinae, Paradoxosomatidae, Strongylosomatidea.
Euporogomphus Hoffman, 2005. Monograph of the Gomphodesmidae, a family of African polydesmoid millipeds. Verlag des Naturhistorischen Museums Wien, 497. M.

Type-species. - E. iboma Hoffman, 2005. Monograph of the Gomphodesmidae, a family of African polydesmoid millipeds. Verlag des Naturhistorischen Museums Wien, 500. Tanzania, Iringa Reg., Njombe Dist., Kipengere Mountains, Iboma Forest; original designation. 1 species.

Gomphodesminae, Gomphodesmidae, Xystodesmoidea, Leptodesmidea.

Exaesiotylus Hoffman, 2005. Monograph of the Gomphodesmidae, a family of African polydesmoid millipeds. Verlag des Naturhistorischen Museums Wien, 490. M.

Type-species. - E. virilis Hoffman, 2005. Monograph of the Gomphodesmidae, a family of African polydesmoid millipeds. Verlag des Naturhistorischen Museums Wien, 494. Democratic Republic of the Congo, Kivu Prov., between Kabare \& Uvira (Luvungi Territory); original designation. 1 species.

Gomphodesminae, Gomphodesmidae, Xystodesmoidea, Leptodesmidea.

Exochopyge Hoffman, 2005. Monograph of the Gomphodesmidae, a family of African polydesmoid millipeds. Verlag des Naturhistorischen Museums Wien, 136. F.

Type-species. - E. spatula Hoffman, 2005. Monograph of the Gomphodesmidae, a family of African polydesmoid millipeds. Verlag des Naturhistorischen Museums Wien, 138. Tanzania, Tanga Reg., Muheza Dist., Amani-Sigi Forest Reserve; original designation. 2 species.

Hapsidodesmini, Marptodesminae, Gomphodesmidae, Xystodesmoidea, Leptodesmidea.

Geniculodesmus Chen, Golovatch et Chang, 2008. Journal of Natural History, 42(37-40): 2548. M.

Type-species. - Habrodesmus inexpectatus Attems, 1944. Zoologischer Anzeiger, 144: 242. Taiwan, Kaohsiung City (rectified from Japan, Tokyo Prefecture, Hachiouji-shi (= Takao) by Chen et al. [2010]); original designation. 1 species.

Chamberliniini, Paradoxosomatinae, Paradoxosomatidae, Strongylosomatidea [Chen et al., 2011b].

Gigantorhacus Jeekel, 2007. Myriapod Memoranda, 10: 55, 93. M.

Type-species. - Platyrhacus mirandus Pocock, 1894. Zoologische Ergebnisse einer Reise in Niederländisch OstIndien, 3: 348. Indonesia, Sumatra, Manindjau, Muka-Muka, Ajer Mantjur; original designation. 5 species.

Platyrhacinae, Platyrhacidae, Platyrhacoidea, Leptodesmidea. 42. M.

Ginglymodesmus Mesibov, 2005. Zootaxa, 1064: 41-

Type-species. - G. tasmanianus Mesibov, 2005. Zootaxa, 1064: 42-46. Australia, Tasmania, Argent River; original designation. 3 species.

Dalodesmidae, Dalodesmidea.

Giryama Hoffman, 2005. Monograph of the Gomphodesmidae, a family of African polydesmoid millipeds. Verlag des Naturhistorischen Museums Wien, 88. F.

Type-species. - G. aruba Hoffman, 2005. Monograph of the Gomphodesmidae, a family of African polydesmoid millipeds. Verlag des Naturhistorischen Museums Wien, 90. 
Kenya, Tsavo East National Park, 30 km (18.8 mi) E Voi, Aruba Lodge; original designation. 1 species.

Elaphogonini, Marptodesminae, Gomphodesmidae, Xystodesmoidea, Leptodesmidea.

Gonatodesmus Golovatch, Geoffroy et VandenSpiegel, 2013. ZooKeys, 414: 48-49. M.

Type-species. - G. communicans Golovatch, Geoffroy et VandenSpiegel, 2013. ZooKeys, 414: 49-52. Vietnam, Dongnai Prov., Cat Tien National Park; original designation. 1 species. dea.

Trichopolydesmidae, Trichopolydesmoidea, Polydesmi-

Graphirhacus Jeekel, 2007. Myriapod Memoranda, 10: 72, 94. M.

Type-species. - Platyrhacus penicillatus Attems, 1914. Archiv für Naturgeschichte, 80A(4): 252. Papua New Guinea (Island of New Guinea); original designation. 1 species.

Psaphodesminae, Platyrhacidae, Platyrhacoidea, Leptodesmidae.

Haematotropis Jeekel, 2000. Myriapod Memoranda, 2: 71-72. M

Type-species. - Polydesmus callipus Peters, 1864 Monatsberichte der Königlichen Preussischen Akademie der Wissenschaften zu Berlin, 1864: 624. Suriname, Mt. Janbasigodo, Upper Gran Rio; Paramaribo, Kabelstation, Coco Plantation, Galibi, Republiek, Paloemen, Tibiti; original designation. 5 species.

Aphelidesmidae, Platyrhacoidea, Leptodesmidea.

Haplogomphodesmus Hoffman, 2005. Monograph of the Gomphodesmidae, a family of African polydesmoid millipeds. Verlag des Naturhistorischen Museums Wien, 449. M.

Type-species. - Gomphodesmus pavani Demange, 1965. Cahiers de la Maboke, 3: 65. Central African Republic, M'Baïki; original designation. 2 species.

Gomphodesminae, Gomphodesmidae, Xystodesmoidea, Leptodesmidea.

*Hapsidodesmus Hoffman, 2005. Monograph of the Gomphodesmidae, a family of African polydesmoid millipeds. Verlag des Naturhistorischen Museums Wien, 128. M.

Type-species. - H. atriclavius Hoffman, 2005. Monograph of the Gomphodesmidae, a family of African polydesmoid millipeds. Verlag des Naturhistorischen Museums Wien, 132. Tanzania, Tanga Region, Lushoto Dist., $16 \mathrm{~km}$ (10 mi) E Lushoto, Mazumbai Estate; original designation. 3 species.

Hapsidodesmini, Marptodesminae, Gomphodesmidae, Xystodesmoidea, Leptodesmidea.

New family-group name: Hapsidodesmini Hoffman, 2005. Monograph of the Gomphodesmidae, a family of African polydesmoid millipeds. Verlag des Naturhistorischen Museums Wien, 121-122. Family-group components: Hapsidodesmus Hoffman, 2005; Protyligmogon Hoffman, 2005; Exochopyge Hoffman, 2005.

Helicodesmus Golovatch, Geoffroy et VandenSpiegel, 2014. ZooKeys, 414: 52. M.

Type-species. - H. anichkini Golovatch, Geoffroy et VandenSpiegel, 2013. ZooKeys, 414: 52-56. Vietnam, Dongnai Prov., Cat Tien National Park; original designation. 1 species.
Trichopolydesmidae, Polydesmidea.

Helictogomphus Hoffman, 2005. Monograph of the Gomphodesmidae, a family of African polydesmoid millipeds. Verlag des Naturhistorischen Museums Wien, 387. M.

Type-species. - Ulodesmus simplex Lawrence, 1953. Annals of the Natal Museum, 12: 301. South Africa, KwaZulu-Natal, Royal Natal National Park, Drakensberg Mountains $50 \mathrm{~km}$ (31.3 mi) W Estacourt, Champagne Castle Hostel; original designation. 1 species.

Ulodesmini, Gomphodesminae, Gomphodesmidae, Xystodesmoidea, Leptodesmidea.

Hoffmanorhacus Shelley et Martínez-Torres, 2013. Zootaxa, 3626(4): 483-484. M.

Type-species. - Proaspis sahlii Jeekel, 1980. Bulletin Zoölogisch Museum Universiteit van Amsterdam, 7(9): 8283. Guadeloupe, Basse-Terre, Parc Naturel de Guadeloupe; original designation. 1 species.

Platyrhacidae, Platyrhacoidea, Leptodesmidea.

*Hoplurorhachis Pocock, 1897. Annals and Magazine of Natural History, 20: 437. F.

Type-species. - H. everettii Pocock, 1897. Annals and Magazine of Natural History, 20: 437-438. NW Borneo (probably Brunei or Malaysia, Eastern Malaysia, Sarawak); original designation. 3 species.

Hoplurorhachini, Platyrhacinae, Platyrhacidae, Platyrhacoidea, Leptodesmidea.

New family-group name: Hoplurorhachini Hoffman, 2001. Revue suisse de Zoologie, 108(2): 410; elevated to subfamilial status by Shelley \& Martínez-Torres [2013]. Family-group components: Hoplurorhachus Pocock, 1897; Creagronopus Hoffman, 2001; Sabarhacus Hoffman, 2001.

Howeosoma Jeekel, 2006. Myriapod Memoranda, 9: 6566. N.

Type-species. - H. carbo Jeekel, 2006. Myriapod Memoranda, 9: 66-69. Australia, New South Wales, Lord Howe Island; original designation. 2 species.

Antichiropodini, Australiosomatinae, Paradoxosomatidae, Strongylosomatidea.

Iemanja Hoffman, 2000. Myriapodologica, 6: 106-107. F.

Type-species. - I. teresa Hoffman, 2000. Myriapodologica, 6: 107-109. Brazil, Espirito Santo, Santa Teresa; original designation. 1 species.

Chelodesminae, Chelodesmidae, Chelodesmoidea, Leptodesmidea.

Inversispina Zhang, in: Zhang, Wang et Zhang, 1997. Invertebrates of Wuling Mountains Area, Southwestern China, 517, 521. F.

Type-species. - I. tortiapicalis Zhang, in: Zhang, Wang et Zhang, 1997. Invertebrates of Wuling Mountains Area, Southwestern China, 517-519, 522. China, Hubei Prov., Hefeng Co. 4 species.

Remark: Omitted from Shelley et al. [2000], Inversispi$n a$ was redefined and rediagnosed by Chen et al. [2011a] and Golovatch [2012, 2013].

Sulciferini, Paradoxosomatinae, Paradoxosomatidae, Strongylosomatidea.

*Ionidesmus Hoffman, 2005. Monograph of the Gomphodesmidae, a family of African polydesmoid millipeds. Verlag des Naturhistorischen Museums Wien, 112. M. 
Type-species. - I. extortus Hoffman, 2005. Monograph of the Gomphodesmidae, a family of African polydesmoid millipeds. Verlag des Naturhistorischen Museums Wien, 112. Tanzania, Tanga Region, Muheza Dist.; original designation. 1 species.

Ionidesmini, Marptodesminae, Gomphodesmidae, Xystodesmoidea, Leptodesmidea.

New family-group name: Ionidesmini Hoffman, 2005. Monograph of the Gomphodesmidae, a family of African polydesmoid millipeds. Verlag des Naturhistorischen Museums Wien, 112. Family-group component: Ionidesmus Hoffman, 2005.

Ithynteria Hoffman, 2005. Monograph of the Gomphodesmidae, a family of African polydesmoid millipeds. Verlag des Naturhistorischen Museums Wien, 444. F.

Type-species. - Ulodesmus pretorianus Lawrence, 1966. Zoologica Africana, 2(2): 253. South Africa, Kruger National Park, Pretoriuskop; original designation. 1 species.

Gomphodesminae, Gomphodesmidae, Xystodesmoidea, Leptodesmidea.

Kainorhacus Jeekel, 2007. Myriapod Memoranda, 10: 57, 93. M.

Type-species. - Platyrhacus mecheli Carl, 1902. Revue suisse de Zoologie, 10: 650. Indonesia, Sumatra, Indragiri; original designation. 1 species.

Platyrhacinae, Platyrhacidae, Platyrhacoidea, Leptodesmidea.

Kilimagomphus Hoffman, 2005. Monograph of the Gomphodesmidae, a family of African polydesmoid millipeds. Verlag des Naturhistorischen Museums Wien, 92. M.

Type-species. - Harmodesmus nitens Attems, 1938. Das Tierreich, 69: 457. Tanzania, Kilimanjaro Reg., Mt. Kilimanjaro, Kibonoto Peak; original designation. 1 species.

Elaphogonini, Marptodesminae, Gomphodesmidae, Xystodesmoidea, Leptodesmidea.

Koponenius Golovatch et VandenSpiegel, 2014. Zootaxa, 3894(1): 142-144. M.

Type-species. - K. unicornis Golovatch et VandenSpiegel, 2014. Zootaxa, 3894(1): 144-148. India, West Bengal, Darjeeling Dist., Kalimpong (Gandhi Ashram); original designation. 3 species.

Haplodesmidae, Haplodesmoidea, Polydesmidea.

Koreadesmus Mikhaljova et Korsós, 2003. Acta Zoologica Academiae Scientiarum Hungaricae, 49(3): 234-235. M.

Type-species. - K. proprius Mikhaljova et Korsós, 2003. Acta Zoologica Academiae Scientiarum Hungaricae, 49(3): 235-238. North Korea, Ryanggang Prov., Konchang; original designation. 1 species.

Remark: Koreadesmus Mikhaljova et Korsós, 2003, is now considered a junior synonym of Cawjeekelia Golovatch, 1980 [Golovatch, 2011].

Sulciferini, Paradoxosomatinae, Paradoxosomatidae, Strongylosomatidea. 6. M.

Leonardesmus Shelley et Shear, 2006. Zootaxa, 1176

Type-species. - L. injucundus Shelley et Shear, 2006. Zootaxa, 1176: 6-14. USA, Washington, Grays Harbor Co., ca. $24 \mathrm{~km}(15 \mathrm{mi})$ NE Aberdeen, along west bank of Middle Fork Satsop R. and $23.2 \mathrm{~km}$ (14.5 mi) E Aberdeen, along east bank of Canyon R.; original designation. 1 species.
Trichopolydesmidae, Trichopolydesmoidea, Polydesmidea.

Leodesmus Mauriès et Geoffroy, 2000. Zoosystema, 22(1): 162-163. M.

Type-species. - Alocodesmus yporangae Schubart, 1946. Livro de Homenagem a R. F. d'Almeida, 37: 308311. Brazil, São Paulo, Areia de Baixo, Gruta de Yporanga; original designation. 1 species.

Cornalatini, Chelodesminae, Chelodesmidae, Chelodesmoidea, Leptodesmidea.

*Leptodesmus DeSaussure, 1859. Linnaea Entomologica, 13: 323. M.

Type-species. - Polydesmus carneus DeSaussure, 1859. Linnaea Entomologica, 13: 324. Brazil; subsequent designation of Pocock [1909: 162]. 21 species in 1980.

Leptodesmini, Chelodesminae, Chelodesmidae, Chelodesmoidea, Leptodesmidea.

New ordinal-group name: Leptodesmidea Brölemann, 1916 (new status from phylum Leptodesmidi proposed by Shelley [2003: 192]). Ordinal-group components: Chelodesmoidea Cook, 1895; Platyrhacoidea Pocock, 1895; Rhachodesmoidea Carl, 1903; Sphaeriodesmoidea Humbert et DeSaussure, 1869; Xystodesmoidea Cook, 1895.

Lippus Özdikmen, 2009. Munis Entomology and Zoology, 4(1): 181-182. M.

Type-species. - Paratylopus strongylosomoides Korsós et Golovatch, 1989. Acta Zoologica Hungarica, 35(3-4): 215-217. Vietnam, Vinh Phu Prov., N of Tam Dao; direct substitution. 1 species.

Remarks: Proposed to replace Paratylopus Korsós et Golovatch, 1989. Acta Zoologica Hungarica, 35(3-4): 215, preoccupied by Paratylopus Matthew, 1904 (Mammalia), Bulletin of the American Museum of Natural History, 20: 211. Because Paratylopus Korsós et Golovatch, 1989, has long been synonymized with Tylopus Jeekel, 1968 [Golovatch, Enghoff, 1993], Lippus Özdikmen, 2009, automatically falls in the same synonymy.

Sulciferini, Paradoxosomatinae, Paradoxosomatidae, Strongylosomatidea.

Lithobiodesmus Hoffman, 2006. Papéis Avulsos de Zoologia, 46(5): 47-48. M.

Type-species. - L. xenoporus Hoffman, 2006. Papéis Avulsos de Zoologia, 46(5): 48-51. Brazil, Rondônia, Nova Esperança; original designation. 1 species.

Chelodesminae, Chelodesmidae, Chelodesmoidea, Leptodesmidea.

Litogonopus Hoffman, 2005. Monograph of the Gomphodesmidae, a family of African polydesmoid millipeds. Verlag des Naturhistorischen Museums Wien, 453. M.

Type-species. - L. mutara Hoffman, 2005. Monograph of the Gomphodesmidae, a family of African polydesmoid millipeds. Verlag des Naturhistorischen Museums Wien, 453. Rwanda, Mutara, Byumba; original designation. 1 species.

Gomphodesminae, Gomphodesmidae, Xystodesmoidea, Leptodesmidea.

Masaigomphus Hoffman, 2005. Monograph of the Gomphodesmidae, a family of African polydesmoid millipeds. Verlag des Naturhistorischen Museums Wien, 256. M.

Type-species. - M. karenae Hoffman, 2005. Monograph of the Gomphodesmidae, a family of African polydes- 
moid millipeds. Verlag des Naturhistorischen Museums Wien, 256. Kenya, Ngong Hills SW of Nairobi; original designation. 1 species.

Aulodesmini, Gomphodesminae, Gomphodesmidae, Xystodesmoidea, Leptodesmidea.

Mastigorhacus Jeekel, 2007. Myriapod Memoranda, 10: 85, 94, 95. M.

Type-species. - Platyrhacus zonatus Carl, 1912. Revue suisse de Zoologie, 20: 149. Indonesia, Kabena Island off southeast coast of Sulawesi; original designation. 2 species. midea.

Erythrhacini, Platyrhacidae, Platyrhacoidea, Leptodes-

*Merodesmus Cook, 1897. Brandtia, 16: 66. M.

Type-species. - Eurydesmus compactilis Gerstäcker, 1873. Die Gliedthier-Fauna des Sansibar-Gebietes. Nach dem von Dr. O. Kersten während der von der Deck'schen Ost-Afrikanischen Expedition im Jahre 1862 und von C. Cooke auf der Insel Sansibar im Jahre 1864 gesammelten Material, Zoologie, 3: 519. Kenya, Mombasa; original designation. 1 species.

Merodesmini, Gomphodesminae, Gomphodesmidae, Xystodesmoidea, Leptodesmidea.

New family-group name: Merodesmini Hoffman, 2005. Monograph of the Gomphodesmidae, a family of African polydesmoid millipeds. Verlag des Naturhistorischen Museums Wien, 260. Family-group component: Merodesmus Cook, 1897.

Mitumbagomphus Hoffman, 2005. Monograph of the Gomphodesmidae, a family of African polydesmoid millipeds. Verlag des Naturhistorischen Museums Wien, 244. M.

Type-species. - Gomphodesmus ferus Attems, 1929. Revue de Zoologie et de Botanique Africaines, 17: 366. Democratic Republic of the Congo, Katanga Prov., Lukonzolwa \& Luanza; original designation. 3 species.

Aulodesmini, Gomphodesminae, Gomphodesmidae, Xystodesmoidea, Leptodesmidea.

Molyrogomphus Hoffman, 2005. Monograph of the Gomphodesmidae, a family of African polydesmoid millipeds. Verlag des Naturhistorischen Museums Wien, 495. M.

Type-species. - Gomphodesmus imbecillus Attems, 1929. Revue de Zoologie et de Botanique Africaines, 17: 370. Democratic Republic of the Congo, Ituri Dist., $25 \mathrm{~km}$ (15.6 mi) SE Mahagi, Niarembi; original designation. 1 species.

Gomphodesminae, Gomphodesmidae, Xystodesmoidea, Leptodesmidea.

Monstrodesmus Golovatch, Geoffroy et VandenSpiegel, 2014. ZooKeys, 414: 56-57. M.

Type-species. - M. flagellifer Golovatch, Geoffroy et VandenSpiegel, 2013. ZooKeys, 414: 57-61. Vietnam, Dongnai Prov., Cat Tien National Park; original designation. 1 species. midea.

Trichopolydesmidae, Trichopolydesmoidea, Polydes-

Nematogomphus Hoffman, 2005. Monograph of the Gomphodesmidae, a family of African polydesmoid millipeds. Verlag des Naturhistorischen Museums Wien, 468. M.

Type-species. - Gomphodesmus micromastus Attems, 1927. Annalen des Naturhistorischen Museums in Wien, 41:
72. Democratic Republic of the Congo, Orientale Prov., Bas-Uele Dist., Angu; original designation. 2 species.

Gomphodesminae, Gomphodesmidae, Xystodesmoidea, Leptodesmidea.

*Neodesmus Cook, 1897. Brandtia, 16: 66. M.

Type-species. - N. juvenis Cook, 1897. Brandtia, 16: 66. Mozambique; original designation. 1 species.

Neodesmini, Gomphodesminae, Gomphodesmidae, Xystodesmoidea, Leptodesmidea.

New family-group name: Neodesmini Hoffman, 2005. Monograph of the Gomphodesmidae, a family of African polydesmoid millipeds. Verlag des Naturhistorischen $\mathrm{Mu}-$ seums Wien, 366. Family-group component: Neodesmus Cook, 1897.

Nevadesmus Shear, 2009. Zootaxa, 2151: 58-59. M.

Type-species. - N. ophimontis Shear, 2009. Zootaxa, 2151: 59-61. USA, Nevada, White Pine Co., Model Cave; original designation; 2 species.

Trichopolydesmidae, Trichopolydesmoidea, Polydesmidea.

Ngurubates Hoffman, 2005. Monograph of the Gomphodesmidae, a family of African polydesmoid millipeds. Verlag des Naturhistorischen Museums Wien, 150. F.

Type-species. - N. emmrichi Hoffman, 2005. Monograph of the Gomphodesmidae, a family of African polydesmoid millipeds. Verlag des Naturhistorischen Museums Wien, 152. Tanzania, Morogoro Dist./Reg., Nuguru Mountains, Dirue River Gorge; original designation. 2 species.

Gomphodesmini, Gomphodesminae, Gomphodesmidae, Xystodesmoidea, Leptodesmidea.

Noteremus Mesibov, 2009. ZooKeys, 7: 56-57. M.

Type-species. - N. summus Mesibov, 2009. ZooKeys, 7: 57-64. Australia, Tasmania, Mt. Weld; original designation. 2 species.

Family unassigned, Dalodesmidea. 66. M.

Notopyrgodesmus Mesibov, 2012. ZooKeys, 217: 65-

Type-species. - N. kulla Mesibov, 2012. ZooKeys, 217: 74-80. Australia, Queensland, $11 \mathrm{~km}(6.9 \mathrm{mi}) \mathrm{W}$ by $\mathrm{N}$ of Bald Hill, McIlwraith Range; original designation. 3 species.

Pyrgodesmidae, Pyrgodesmoidea, Polydesmidea. 81. F.

Ochrotropis Jeekel, 2000. Myriapod Memoranda, 2:

Type-species. - Aphelidesmus guianensis Chamberlin, 1923. Occasional Papers of the Museum of Zoology, University of Michigan, 133: 46. Guyana, Demerara, Sand Hill Forest; original designation. 2 species.

Aphelidesmidae, Platyrhacoidea, Leptodesmidea.

Orocladosoma Jeekel, 2000. Myriapod Memoranda, 2: 39-40. N.

Type-species. - Australiosoma kosciuskovagum Brölemann, 1913. Records of the Australian Museum, 10: 100. Australia, New South Wales, Pretty Point, Mt. Kosciusko; original designation. 1 species.

Australiosomatini, Australiosomatinae, Paradoxosomatidae, Strongylosomatidea. 
Orthomorphoides Likhitrakarn, Golovatch et Panha, 2011. ZooKeys, 131: 144. M.

Type-species. - Orthomorpha setosa Attems, 1937. Das Tierreich, 68: 71. Vietnam, Lamdong Prov., Dalat; original designation. 2 species.

Orthomorphini, Paradoxosomatinae, Paradoxosomatidae, Strongylosomatidea. 6. M.

Pantanalodesmus Hoffman, 2000. Myriapodologica, 7:

Type-species. - P. marinezae Hoffman, 2000. Myriapodologica, 7: 6-8. Brazil, Mato Grosso, Fazenda Ipiranga, km 10 on Transpanatal Highway near Poconé; original designation. 1 species.

Telonychopodini, Chelodesminae, Chelodesmidae, Chelodesmoidea, Leptodesmidea

*Paradoxosoma Daday, 1889. Természetrajzi Füzetek, 12: $135 . \mathrm{N}$.

Type-species. - Paradoxosoma granulatum Daday, 1889. Természetrajzi Füzetek, 12: 135. Greece; monotypy. $\sim 14$ species as of 1980 .

Remark: Paradoxosoma Daday, 1889, is now considered a synonym of Stosatea Gray, 1843.

Paradoxosomatini, Paradoxosomatinae, Paradoxosomatidae, Strongylosomatidea.

New ordinal-group name: Paradoxosomatidea Hoffman, 1967. Revue de Zoologie et de Botanique Africaines, 76 202, now considered a synonym of Strongylosomatidea Brölemann, 1916. Ordinal-group component: Paradoxosomatidae Daday, 1889

Remark: Omitted from Shelley et al. [2000].

Paredrodesmus Mesibov, 2003. Zootaxa, 368: 8-9. M. Type-species. - P. taurulus Mesibov, 2003. Zootaxa, 368: 9-11. Australia, Tasmania, Tarraleah; original designation. 6 species.

Family not assigned, Dalodesmidea.

Perittogonopus Jeekel, 2003. Myriapod Memoranda, 6: 41-42. M.

Type-species. - P. perplexus Jeekel, 2003. Myriapod Memoranda, 6: 42-45. Australia, New South Wales, Wauchope, Mt. Boss State Forest. Monotypy. 1 species.

Australiosomatini, Australiosomatinae, Paradoxosomatidae. Strongylosomatidea.

Plectrogonodesmus Hoffman, 2012. Papéis Avulsos de Zoologia, 52(8): 104. M.

Type-species. - Leptodesmus gounellei Brölemann, 1903. Annales de la Société Entomologique de France, 71: 665. Brazil, Bahia, Condeúba (= Santo Antonio da Barra); original designation. 1 species.

Chelodesminae, Chelodesmidae, Chelodesmoidea, Leptodesmidea

Pogoro Hoffman, 2005. Monograph of the Gomphodesmidae, a family of African polydesmoid millipeds. Verlag des Naturhistorischen Museums Wien, 169. M.

Type-species. - P. scharffi Hoffman, 2005. Monograph of the Gomphodesmidae, a family of African polydesmoid millipeds. Verlag des Naturhistorischen Museums Wien, 169. Tanzania, Iringa Reg., Mufindi Dist., Udzungwa Scarp Forest Reserve above Chita; original designation. 1 species.

Gomphodesmini, Gomphodesminae, Gomphodesmidae, Xystodesmoidea, Leptodesmidea.
Praeterpediculus Vohland, in: Vohland et Hamer, 2013. African Invertebrates, 549(1): 264-265. M.

Type-species. - Phaeodesmus niger Attems, 1928. Annals of the South African Museum, 26: 249. Namibia, Namutoni; original designation. 1 species.

Cnemodesmini, Paradoxosomatinae, Paradoxosomatidae, Strongylosomatidea.

Pratherodesmus Shear, 2009. Zootaxa, 2151: 51. M.

Type-species. - P. voylesi Shear, 2009. Zootaxa, 2151: 51-54. USA, Arizona, Mojave Co., Millipede Cave; original designation. 3 species.

Trichopolydesmidae, Trichopolydesmoidea, Polydesmidea.

Proagomphus Hoffman, 2005. Monograph of the Gomphodesmidae, a family of African polydesmoid millipeds. Verlag des Naturhistorischen Museums Wien, 502. M.

Type-species. - Gomphodesmus mansuetus Attems, 1953. Annales du Musée Royal du Congo Belge, Série $8^{\circ}$, Sciences Zoologiques, 18: 43. Democratic Republic of the Congo, Équateur Prov., Ubangi Dist., Budjala-Batangu; original designation. 1 species.

Gomphodesminae, Gomphodesmidae, Xystodesmoidea, Leptodesmidea.

Procophorella Mesibov, 2003. Zootaxa, 368: 2-3. F.

Type-species. - P. innupta Mesibov, 2003. Zootaxa, 368: 3-7. Australia, Tasmania, Arm River; original designation. 2 species.

Family not assigned, Dalodesmidea.

Protastrodesmus Hoffman, 2005. Monograph of the Gomphodesmidae, a family of African polydesmoid millipeds. Verlag des Naturhistorischen Museums Wien, 344. M.

Type-species. $-P$. planus Hoffman, 2005. Monograph of the Gomphodesmidae, a family of African polydesmoid millipeds. Verlag des Naturhistorischen Museums Wien, 345. Tanzania, Tanga Reg., Umba River; original designation. 1 species.

Astrodesmini, Gomphodesminae, Gomphodesmidae, Xystodesmoidea, Leptodesmidea.

Protyligmagon Hoffman, 2005. Monograph of the Gomphodesmidae, a family of African polydesmoid millipeds. Verlag des Naturhistorischen Museums Wien, 122. M.

Type-species. - P. kanga Hoffman, 2005. Monograph of the Gomphodesmidae, a family of African polydesmoid millipeds. Verlag des Naturhistorischen Museums Wien, 124. Tanzania, Morogoro Dist./Reg., Kanga Mountains, Kanga Forest Reserve; original designation. 2 species.

Hapsidodesmini, Marptodesminae, Gomphodesmidae, Xystodesmoidea, Leptodesmidea.

Pseudoporatia Golovatch, 2000. Amazoniana, 15(3/4): 228. F.

Type-species. - P. perplexa Golovatch, 2000. Amazoniana, 15(3/4): 229. Brazil, Amazônas, environs of Manaus, Reserva Florestal A. Ducke; original designation. 1 species.

Remark: The printed date is December 1999, but the actual publication date was April 2000 (Adis, in litt.).

Pyrgodesmidae, Pyrgodesmoidea, Polydesmidea.

Pseudosundanina Nguyen, 2010. Zootaxa, 2479: 60. F. Type-species. - Sundanina falcata Attems, 1937. Das Tierreich, 68: 161-162. Vietnam, Dalat; original designation. 1 species. 
Sundaninini, Paradoxosomatinae, Paradoxosomatidae, Strongylosomatidea.

Retrorsia Shelley, 2003. Zootaxa, 296: 3-5. F.

Type-species. - $R$. leonardi Shelley, 2003. Zootaxa, 296: 5-7. USA, Oregon, Multnomah Co., Columbia River Gorge National Scenic Area, Ainsworth State Park; original designation. 2 species.

Polydesmidae, Polydesmoidea, Polydesmidea.

Rhicnostethus Hoffman, 2006. Papéis Avulsos de Zoologia, 46(5): 51-52. M.

Type-species. - R. rondoni Hoffman, 2006. Papéis Avulsos de Zoologia, 46(5): 52-55. Brazil, Rondônia, Nova Esperança; original designation; 2 species.

Chelodesminae, Chelodesmidae, Chelodesmoidea, Leptodesmidea.

Rondonaria Hoffman, 2006. Papéis Avulsos de Zoologia, 46(5): 44. F.

Type-species. - R. schubarti Hoffman, 2006. Papéis Avulsos de Zoologia, 46(5): 44-47. Brazil, Rondônia, Santa Cruz da Serra; original designation. 1 species.

Chelodesminae, Chelodesmidae, Chelodesmoidea, Leptodesmidea.

Sabarhacus Hoffman, 2001. Revue suisse de Zoologie, 108(2): 417, 419. M.

Type-species. - S. derodontus Hoffman, 2001. Revue suisse de Zoologie, 108(2): 420-423. Malaysia, East Malaysia, Sabah; original designation. 2 species.

Hoplurorhachini, Platyrhacinae, Platyrhacidae, Platyrhacoidea, Leptodesmidea.

Sapamorpha Golovatch, 2009. Arthropoda Selecta, 18(34): 122-123. F.

Type-species. - S. complexa Golovatch, 2009. Arthropoda Selecta, 18(3-4): 123-124. Vietnam, Lao Cai Prov., Hoang Lien National Park west of Sapa; original designation. 1 species.

Orthomorphini, Paradoxosomatinae, Paradoxosomatidae, Strongylosomatidea.

Scaptogonodesmus Hoffman, 2005. Monograph of the Gomphodesmidae, a family of African polydesmoid millipeds. Verlag des Naturhistorischen Museums Wien, 350. M.

Type-species. - S. gedi Hoffman, 2005. Monograph of the Gomphodesmidae, a family of African polydesmoid millipeds. Verlag des Naturhistorischen Museums Wien, 350. Kenya, $20 \mathrm{~km}$ (12.5 mi) SW Malindi, Gedi ruins at Watamu; original designation. 1 species.

Astrodesmini, Gomphodesminae, Gomphodesmidae, Xystodesmoidea, Leptodesmidea.

*Schizogomphodesmus Brolemann, 1920. Voyage de Ch. Alluaud et R. Jeannel en Afrique orientale, Myriapoda, 3: 209. M.

Type-species. - S. longispina Brolemann, 1920. Voyage de Ch. Alluaud et R. Jeannel en Afrique orientale, Myriapoda 3: 210 . Kenya, Naivasha, Rift Valley; original designation. 1 species.

Schizogomphodesmini, Marptodesminae, Gomphodesmidae, Xystodesmoidea, Leptodesmidea.

New family-group name: Schizogomphodesmini Hoffman, 2005. Monograph of the Gomphodesmidae, a family of
African polydesmoid millipeds. Verlag des Naturhistorischen Museums Wien, 115. Family-group component: Schizogomphodesmus Brolemann, 1920.

Sellanucheza Enghoff, Golovatch et Nguyen, 2004. Arthropoda Selecta, 13(1-2): 39. F.

Type-species. - Szechuanella tenebra Hoffman, 1960. Annals and Magazine of Natural History, Series 13, 3: 535538; direct substitution as Szechuanella Hoffman, 1960, is preoccupied by Szechuanella Lu, 1959 (Trilobita). 6 species.

Remarks: Özdikmen [2007] proposed Cemsunguria Özdikmen, 2007, nom.nov., to replace Szechuanella Hoffman, 1960, but this name is a junior objective synonym of Sellanucheza Enghoff, Golovatch et Nguyen, 2004.

Tonkinosomatini, Paradoxosomatinae, Paradoxosomatidae, Strongylosomatidea.

Sequoiadesmus Shear et Shelley, 2008. Zootaxa, 1693: 43. M

Type-species. - S. krejcae Shear et Shelley, 2008. Zootaxa, 1693: 43-47. USA, California, Tulare Co., Sequoia National Park, Hurricane Crawl Cave; original designation. 1 species.

Trichopolydesmidae, Trichopolydesmoidea, Polydesmidea.

Setoisenoton Mesibov, 2010. Zootaxa, 2571: 54. M.

Type-species. - S. pallidus Mesibov, 2010. Zootaxa, 2571: 54-58. Australia, Tasmania, $5 \mathrm{~km}$ (3.1 mi) S Renison Bell; original designation. 1 species.

Family not assigned, Dalodesmidea.

*Sigodesmus Cook, 1897. Brandtia, 16: 67. M.

Type-species. - S. indigus Cook, 1897. Brandtia, 16: 67. Kenya, Witu; original designation. 5 species.

Sigodesmini, Gomphodesminae, Gomphodesmidae, Xystodesmoidea, Leptodesmidea.

New family-group name: Sigodesmini Hoffman, 2005. Monograph of the Gomphodesmidae, a family of African polydesmoid millipeds. Verlag des Naturhistorischen Museums Wien, 353. Family-group component: Sigodesmus Cook, 1897.

Silvattia Jeekel, 2009. Myriapod Memoranda, 11: 77. F. Type-species. - Atropisoma horvathi Silvestri, 1899. Természetrajzi Füzetek, 22: 207. Papua New Guinea, Madang Prov., Eriba, Astrolabe Bay; original designation. 5 species.

Eustrongylosomatini, Paradoxosomatinae, Paradoxosomatidae, Strongylosomatidea.

Simplogonomorpha Nguyen et Korsós, 2011. ZooKeys, 156: 31-32. F.

Type-species. - Haplogonosoma falcatum Attems, 1953. Mémoires du Muséum National d'Histoire Naturelle, Paris \{N. S., Sér. A, Zool.\}, 5(3): 177. Laos, Xiangkhouang (= Xieng Kuang) Prov.; original designation. 1 species.

Chamberliniini, Paradoxosomatinae, Paradoxosomatidae, Strongylosomatidea.

Sinomorpha Golovatch, 2013. Arthropoda Selecta, 22(1): 24-26. F.

Type-species. - S. setosa Golovatch, 2013. Arthropoda Selecta, 22(1): 26-28. China, Sichuan, Mt. Emei Shan, Wannian Monastery; original designation. 1 species.

Orthomorphini, Paradoxosomatinae, Paradoxosomatidae, Strongylosomatidea. 
Snoqualmia Shear, 2012. Insecta Mundi, 0238: 6-8. F. Type-species. - S. snoqualmie Shear, 2012. Insecta Mundi, 0238: 8-10. USA, Washington, King Co., Twin Falls/Iron Horse trailhead; original designation. 2 species.

Polydesmidae, Polydesmoidea, Polydesmidea.

Solentanodesmus Antić et Reip, in: Antić, Reip, Dražina, Rađa et Makarov, 2014. Zootaxa, 3884(2): 106-107. M.

Type-species. - S. insularis Antić et Reip, in: Antić, Reip, Dražina, Rađa et Makarov, 2014. Zootaxa, 3884(2): 107-109. Croatia, Šolta Island, Grohote, Jama na Benkotovu Pit; original designation. 1 species.

Trichopolydesmidae, Trichopolydesmoidea, Polydesmidea.

Stenotyligma Hoffman, 2005. Monograph of the Gomphodesmidae, a family of African polydesmoid millipeds. Verlag des Naturhistorischen Museums Wien, 459. F

Type-species. - S. nasmodes Hoffman, 2005. Monograph of the Gomphodesmidae, a family of African polydesmoid millipeds. Verlag des Naturhistorischen Museums Wien, 461. Tanzania, Mpwapwa Dist., 50 km (31 mi) S Mpwapwa, Ruhebo Mountains, Chugu Mountain; original designation. 1 species.

Gomphodesminae, Gomphodesmidae, Xystodesmoidea, Leptodesmidea.

Streptelopus Hoffman, 2005. Monograph of the Gomphodesmidae, a family of African polydesmoid millipeds. Verlag des Naturhistorischen Museums Wien, 238. M.

Type-species. - Gomphodesmus asperulus Attems, 1929. Revue de Zoologie et de Botanique Africaines, 30: 66. Democratic Republic of the Congo, Katanga Prov., Kambaye (lectotype locality); original designation. 3 species.

Aulodesmini, Gomphodesminae, Gomphodesmidae, Xystodesmoidea, Leptodesmidea.

*Strongylosoma Brandt, 1833. Bulletin de la Société Impériale des Naturalistes de Moscou, 6: 205. N.

Type-species. - Julus stigmatosus Eichwald, 1830. Zoologia Specialis, 2: 124 (= Strongylosoma iuloides Brandt, 1833, Bulletin de la Société Impériale des Naturalistes de Moscou, 6: 205. Lithuania/Ukraine); monotypy. 10 acknowledged species in 1980 .

Paradoxosomatini, Paradoxosomatinae, Paradoxosomatidae, Stronglyosomatidea.

New ordinal-group name: Strongylosomatidea Brölemann, 1916 (new status from phylum Strongylosomini (recte: Strongylosomatini) proposed by Shelley [2003: 192]. Ordinal-group component: Paradoxosomatidae Daday, 1889.

Tauricollarium Golovatch, 2000. Amazoniana, 15(3/4): 225. N.

Type-species. - T. biramosum Golovatch, 2000. Amazoniana, 15(3/4): 226. Brazil, Amazônas, environs of Manaus, Reserva Florestal A. Ducke; original designation. 2 species.

Remark: The printed date is December 1999, but the actual publication date was April 2000 (Adis, in litt.).

Pyrgodesmidae, Pyrgodesmoidea, Polydesmidea.

Tessarorhacus Jeekel, 2007. Myriapod Memoranda, 10: 77, 84. M.

Type-species. - Platyrhacus paliger Attems, 1914. Archiv für Naturgeschichte, 80A(4): 252. Papua New Guinea (Island of New Guinea), Waigeu, Kaiawat, Beo, Bajon; original designation. 5 species.
Psaphodesminae, Platyrhacidae, Platyrhacoidea, Leptodesmidea.

Tholerosoma Mesibov, 2006. Zootaxa, 1354: 37. N. Type-species. - T. monteithi Mesibov, 2006. Zootaxa, 1354: 38-42. Australia, Queensland, Palmerston National Park, Downey Creek Road; original designation. 2 species.

Paradoxosomatidae, Strongylosomatidea.

Remark. Neither tribal nor subfamilial positions are indicated in the original description.

Trematorhacus Jeekel, 2007. Myriapod Memoranda, 10: 67, 94. M.

Type-species. - Platyrhacus coelebs Carl, 1902. Revue suisse de Zoologie, 10: 644. Indonesia, Sumatra; original designation. 1 species. midea.

Platyrhacinae, Platyrhacidae, Platyrhacoidea, Leptodes-

Trilobodesmus Golovatch et Mauriès, 2007. Journal of Afrotropical Zoology, 3: 11-13. M.

Type-species. - T. levequei Golovatch et Mauriès, 2007. Journal of Afrotropical Zoology, 3: 13-14; Yemen, Sokotra Island, Hoq Cave; original designation. 1 species. dea.

Trichopolydesmidae, Trichopolydesmoidea, Polydesmi-

*Tycodesmus Cook, 1896. Proceedings of the United States National Museum, 18: 83. M.

Type-species. - T. medius Cook, 1896. Proceedings of the United States National Museum, 18: 83; type locality not provided originally, but according to Cook [1899: 700] it is Tanzania, Dodoma Reg., Mpapua (= German East Africa, Mpapua); monotypy. 3 species.

Tycodesmini, Gomphodesminae, Gomphodesmidae, Xystodesmoidea, Leptodesmidea.

New family-group name: Tycodesmini Hoffman, 2005. Monograph of the Gomphodesmidae, a family of African polydesmoid millipeds. Verlag des Naturhistorischen Museums Wien, 266. Family-group component: Tycodesmus Cook, 1896.

*Ulodesmus Cook, 1897. Brandtia, 16: 66. M.

Type-species. - U. micramma Cook, 1897. Brandtia, 16: 66. South Africa, KwaZulu-Natal, Durban and Maritzburg (= ?Pietermaritzburg); original designation. 26 species.

Ulodesmini, Gomphodesminae, Gomphodesmidae, Xystodesmoidea, Leptodesmidea.

New family-group name: Ulodesmini Hoffman, 2005. Monograph of the Gomphodesmidae, a family of African polydesmoid millipeds. Verlag des Naturhistorischen Museums Wien, 378. Family-group components: Ulodesmus Cook, 1897; Antiphonus Attems, 1901; Helictogomphus Hoffman, 2005 .

Umbridesmus Vohland, in: Vohland et Hamer, 2013. African Invertebrates, 54(1): 268. M.

Type-species. - Pagioprium millequingentesimum Attems, 1944. Zoologischer Anzeiger, 144(11/12): 268-269. Namibia, Outjo; original designation. 1 species.

Paradoxosomatinae, Paradoxosomatidae, Strongylosomatidea.

Vaalogomphus Hoffman, 2005. Monograph of the Gomphodesmidae, a family of African polydesmoid millipeds. Verlag des Naturhistorischen Museums Wien, 446. M. 
Type-species. - Ulodesmus macrodontus Lawrence, 1966. Zoologica Africana, 2: 251. South Africa, Kruger National Park, Klawerpan; original designation. 1 species.

Gomphodesminae, Gomphodesmidae, Xystodesmoidea, Leptodesmidea.

Vanzolegulus Hoffman, 2002. Myriapodologica, 7(12): 115-116. M.

Type-species. - V. limbatus Hoffman, 2002. Myriapodologica, 7(12): 116-117. Brazil, Mato Grosso, Vila Bela da Santissima Trinidade; monotypy. 1 species.

Telonychopodini, Chelodesminae, Chelodesmidae, Chelodesmoidea, Leptodesmidea.

Velebitodesmus Antić et Reip, in: Antić, Reip, Dražina, Rađa et Makarov, 2014. Zootaxa, 3884(2): 109-110. M.

Type-species. - V. cavernicolus Antić et Reip, in: Antić, Reip, Dražina, Rađa et Makarov, 2014. Zootaxa, 3884(2): 114-117. Croatia, Mt. Velebit, Gračae, Crnopac, Manižaba Pit; original designation. 1 species.

Trichopolydesmidae, Trichopolydesmoidea, Polydesmidea.

Victoriombrus Mesibov, 2004. Memoirs of Museum Victoria, 61(1): 41-43. M.

Type-species. - V. acanthus Mesibov, 2004. Memoirs of Museum Victoria, 61(1): 43-45. Australia, Victoria, Young Creek Road, $0.2 \mathrm{~km}(0.1 \mathrm{mi})$ NE Ciancio Creek crossing; original designation. 2 species.

Dalodesmidae, Dalodesmidea.

Vigilia Özdikmen, 2009. Munis Entomology and Zoology, 4(1): 180-181. M.

Type-species. - Curimagua granulata Hoffman, 1982. Journal of Natural History, 16: 646-648. Venezuela, Falcon Edo., Serrania de San Luis, Valle de Curimagua, Cueva de Camburales; direct substitution. 1 species.

Remarks: Proposed to replace Curimagua Hoffman, 1982, Journal of Natural History, 16: 645, preoccupied by Curimagua Forster et Platnick, 1976 (Araneae), American Museum Novitates, 2619: 24. Vigilia Özdikmen, 2009, nom. nov., is actually a junior objective synonym of Curimaguana Hoffman, 2007, nom. nov., which has two years of priority for the replacement name.

Batodesmini, Chelodesminae, Chelodesmidae, Chelodesmoidea, Leptodesmidea.

Virungula Hoffman, 2005. Monograph of the Gomphodesmidae, a family of African polydesmoid millipeds. Verlag des Naturhistorischen Museums Wien, 461. F

Type-species. - Gomphodesmus conifer Attems, 1937. Revue de Zoologie et de Botanique Africaines, 30: 68. Democratic Republic of the Congo, Ngoma, N. Kivu; original designation. 1 species.

Gomphodesminae, Gomphodesmidae, Xystodesmoidea, Leptodesmidea.

Wulingina Zhang, in: Zhang, Wang et Zhang, 1997. Invertebrates of Wuling Mountains Area, Southwestern China, 514, 520-521. F.

Type-species. - W. miniloba Zhang, in: Zhang, Wang et Zhang, 1997. Invertebrates of Wuling Mountains Area, Southwestern China, 517-519, 522. China, Hubei Prov., Hefeng Co. 2 species.

Remark. Omitted from Shelley et al. [2000].
Paradoxosomatinae, Paradoxosomatidae, Strongylosomatidea.

ACKNOWLEDGEMENTS. We gratefully acknowledge the pre-publication review of T. Wesener (Zoological Research Museum Alexander Koenig, Leibniz Institute for Animal Biodiversity, Bonn, Germany). Special thanks go to J.P. Mauriès (Muséum national d'Histoire naturelle, Paris, France) and R. Mesibov (Penguin, Tasmania, Australia) for spotting a number of errors in the Nomenclator II. In addition, A. Minelli (University of Padua, Italy) kindly helped us establish the gender of a few recently proposed genera.

\section{References}

Bollman C.H. 1887. Notes on North American Julidae // Annals of the New York Academy of Sciences. Vol.4. P.25-44.

Brandt J.F. 1841. Generis Juli specierum enumeratio, adjectis plurium, quae hucusque nondum innotuerunt specierum brevibus descriptionibus ad Musei Academiae Scientiarum Petropolitanae specimina factus // Bulletin scientifique publié par l'Académie Impériale des Sciences de Saint-Pétersbourg. T.8. Nos 7-8. P.97-128 (for 1840)

Chen C.c., Golovatch S.I., Mikhaljova E.V., Chang H.w. 2010. The millipede genus Anoplodesmus Pocock, 1895, recorded in Taiwan for the first time, with descriptions of two new species (Diplopoda: Polydesmida: Paradoxosomatidae: Sulciferini) // Zootaxa. Vol.2399. P.20-30.

Chen C.c., Golovatch S.I., Mikhaljova E.V., Chang H.w. 2011a. The genus Inversispina Zhang, in Zhang, Wang et Zhang, 1997, new to the millipede fauna of Taiwan, with the description of a new species (Diplopoda: Polydesmida: Paradoxosomatidae: Alogolykinae) // Arthropoda Selecta. Vol.20. No.4. P.251-257.

Chen C.c., Golovatch S.I., Chang H.w, Chen S.h. 2011b. Revision of the Taiwanese millipede genus Chamberlinius Wang, 1956, with descriptions of two new species and a reclassification of the tribe Chamberlinini (Diplopoda, Polydesmida, Paradoxosomatidae, Paradoxosomatinae) // ZooKeys. Vol.98. P.1-27.

Cook O.F. 1895. Introductory note on the families of Diplopoda // Cook O.F., Collins G.N. The Craspedosomatidae of North America. Annals of the New York Academy of Science. Vol.9. P.1-9.

Cook O.F. 1899. African Diplopoda of the family Gomphodesmidae // Proceedings of the United States National Museum. Vol.21. P.677-739.

Gervais P. 1847. Myriapodes // Walckenaer D.A., Gervais P. (eds.). Histoire naturelle des Insectes. Aptères. Paris, Roret. T.4. P.xvi+1-333.

Golovatch S.I. 2011. On several new or poorly-known Oriental Paradoxosomatidae (Diplopoda: Polydesmida), XI // Arthropoda Selecta. Vol.20. No.4. P.259-266.

Golovatch S.I. 2012. On several new or poorly-known Oriental Paradoxosomatidae (Diplopoda: Polydesmida), XII // Arthropoda Selecta. Vol.21. No.1. P.1-12.

Golovatch S.I. 2013. On several new or poorly-known Oriental Paradoxosomatidae (Diplopoda: Polydesmida), XIII // Arthropoda Selecta. Vol.22. No.4. P.307-332.

Golovatch S.I., Enghoff H. 1993. Review of the millipede genus Tylopus, with descriptions of new species from Thailand (Diplopoda, Polydesmida, Paradoxosomatidae) // Steenstrupia. Vol.19. No.3. P.85-125.

Golovatch S.I., Li Y.b., Liu W.x., Geoffroy J.-J. 2012. Three new cavernicolous species of dragon millipedes, genus Desmoxytes Chamberlin, 1923, from southern China, with notes on a formal congener from the Philippines (Diplopoda, Polydesmida, Paradoxosomatidae) // ZooKeys. Vol.185. P.1-17.

Hannibal J. 2000. Hexecontasoma, a new helminthomorph millipede (Hexecontasomatidae n. fam.) from the Mazon Creek, Illinois, fauna (Carboniferous, North America // Fragmenta Faunistica. Vol.43 (Supplement). P.19-35. 
Hoffman R.L. 1980. Classification of the Diplopoda. Genève: Muséum d'histoire naturelle. 237 pp. (for 1979).

Hoffman R.L. 1999. Checklist of the millipeds of North and Middle America // Virginia Museum of Natural History Special Publication Number 8. 584 pp.

Jeekel C.A.W. 1971. Nomenclator generum et familiarum Diplopodorum: A list of the genus and family-group names in the class Diplopoda from the 10th edition of Linnaeus, 1758, to the end of 1957 // Monografieën van de Nederlandse Entomologische Vereniging. Vol.5. P.i-xii+1-412.

Marek P.E., Bond J.E., Sierwald P. 2003. Rhinocricidae Systematics II: A species catalog of the Rhinocricidae (Diplopoda: Spirobolida) with synonymies // Zootaxa. Vol.308. P.1-108.

Mauriès J.-P. 1983. Cambalides nouveaux et peu connus d'Asie, d'Amérique et d'Océanie. I. Cambalidae et Cambalopsidae // Bulletin du Muséum National d'Histoire Naturelle, série 4, section A, Zoologie. T.5. Fasc.1. P.247-276.

Mauriès J.-P. 1987. Cambalides nouveaux et peu connus d'Asie, d'Amérique et d'Océanie. II. Pseudonannolenidae, Choctellidae // Bulletin du Muséum National d'Histoire Naturelle, Paris, série 4, section A, Zoologie. T.9. Fasc.1. P.169-199.

Mauriès J.-P. 2013. Trois espèces nouvelles de diplopodes cavernicoles de l'Andalousie (Espagne) (Diplopoda: Polydesmida, Polydesmidae; Chordeumatida: Vandeleumatidae, Opisthocheiridae) // Arthropoda Selecta. Vol.22. No.2. P.97-112.

Özdikmen H. 2007. A nomenclatural act on Myriapoda: replacement names for homonymous generic names of a millipede and a centipede (Myriapoda: Diplopoda and Chilopoda) // Munis Entomology and Zoology. Vol.2. No.2. P.433-435.

Özdikmen H. 2008. New family and genus names, Kirkayakidae nom. nov., and Kirkayakus nom. nov., for the millipedes (Diplopoda: Chordeumatida) // Munis Entomology and Zoology. Vol.3. No.1. P.342-344.

Özdikmen H. 2009. Substitute names for four preoccupied millipede genera (Diplopoda) // Munis Entomology and Zoology. Vol.4. No.1. P.180-183.

Pedroli-Christen A., Mauriès J.-P. 1992. Un genre oublié d'Atractosomatinae des Alpes italo-suisses, Bomogona Cook, 1895: statut, contenu, chorologie (Diplopoda, Craspedosomatida) // Bulletin du Muséum national d'Histoire naturelle, $4^{\text {ème }}$ sér., Section A, No.2. T.14. P.457-472.

Pérez-Asso A.R. 2002. Cylindromus, un nuevo nombre sinónimo del género Ricodesmus (Diplopoda: Polydesmida: Chelodesmidae) // Solenodon. Vol.2. P.27-30.

Pitz K.M., Sierwald P. 2010. Phylogeny of the millipede order Spirobolida (Arthropoda: Diplopoda: Helminthomorpha) // Cladistics. Vol.26. P.497-525.

Pocock R.I. 1894. Contributions to our knowledge of the arthropod fauna of the West Indies. - Part III. Diplopoda and Malacopoda, with a supplement on the Arachnida of the class Pedipalp // Linnean Journal of Zoology, London. Vol.24. P.473-544.
Pocock R.I. 1909. Diplopoda // Biologia Centrali-Americana. London. Taylor \& Francis. P.162.

Shear W.A. 2011. Class Diplopoda de Blainville in Gervais, 1844 // Zhang Z.-Q. (ed.). Animal biodiversity: An outline of higher-level classification and survey of taxonomic richness. Zootaxa. Vol.3148. P.159-164.

Shelley R.M. 2003. A revised, annotated, family-level classification of the Diplopoda // Arthropoda Selecta. Vol.11 (for 2002). No.3. P.187-207.

Shelley R.M. 2007. Taxonomy of extant Diplopoda (Millipeds) in the modern era: Perspectives for future advancements and observations on the global diplopod community (Arthropoda: Diplopoda) // Zhang Z.-Q., Shear W.A. (eds.). Linnaeus Tercentenary: Progress in Invertebrate Taxonomy. Zootaxa. Vol.1668. P.1-766.

Shelley R.M., Martínez-Torres D. 2013. The milliped family Platyrhacidae (Polydesmida: Leptodesmidea) in the West Indies: Proposal of Hoffmanorhacus n. gen.; description and illustrations of males of Proaspis aitia Loomis, 1941; redescription of Nannorrhacus luciae (Pocock, 1894); hypotheses on origins and affinities; and an updated New World familial distribution // Zootaxa. Vol.3626. No.4. P.477-498.

Shelley R.M., Sierwald P., Kiser S.B., Golovatch S.I. 2000. Nomenclator generum et familiarum Diplopodorum II. A list of the genus and family-group names in the class Diplopoda from 1958 through 1999. Sofia: Pensoft Publishers. 167 p.

Silvestri F. 1896. I diplopodi. Parte I. Sistematica // Annali del Museo Civico di Storia Naturale di Genova. Ser.2. T.36. P.121-254.

Stoev P., Geoffroy J.-J. 2004. Review of the millipede family Paracortinidae Wang \& Zhang 1993 (Diplopoda: Callipodida) // Acta Arachnologica. Vol.53. No.2. P.93-103.

Verhoeff K.W. 1909. Über die Vulven der Ascospermophora, das Cyphopodensegment und Spermatophoren als Begattungszeichen // Sitzungsberichte der Gesellschaft Naturforschender Freunde zu Berlin. Jg.1909. S.209-219.

Wesener T. 2009. Unexplored richness: discovery of 31 new species of giant pill-millipedes endemic to Madagascar, with a special emphasis on microendemism (Diplopoda, Sphaerotheriida) // Zootaxa. Vol.2097. P.1-134.

Wesener T. 2014. A new phylogenetic analysis of the Sphaerotheriida (Diplopoda) with a revision of the Australian giant pill-millipedes // Invertebrate Systematics. Vol.28. P.196-213.

Wilson H.M. 2005a. A new genus of archipolypodan millipede from the Coseley Lagerstätte, Upper Carboniferous, UK // Palaeontology. Vol.48. Part 5. P.1097-1100.

Wilson H.M. 2005b. Zosterogrammida, a new order of millipedes from the Middle Silurian of Scotland and the Upper Carboniferous of Euramerica // Palaeontology. Vol.48. Part 5. P.11011110.

Wood H.C. 1865. The Myriapoda of North America // Transactions of the American Philosophical Society. Vol.13. P.137-248.

Responsible editor K.G. Mikhailov

\section{INDEX}

acanthus, Victoriombrus .................... 22 Acerhacus ........................................ 11 acmophorus, Platyrhacus; type-species of Clastrorhacus ...................................... 13 acrotrifoliolatus, Agaricogonopus ........ 6 Adisiella ......................................... 11

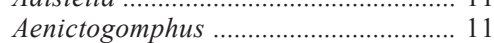
Aethalosoma .......................................... 11 Afraustraloxenodes .............................. 3 Agaricogonopus ...................................... 6 Agrophogonus ...................................... 11 Alienostreptus ......................................... 6 alienus, Thyropygus; type-species of Alienostreptus ........................................ 6 Alluviobolus ........................................ 5
Altajella ..................................... 2, 8, 9

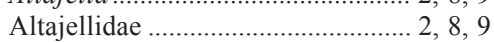
Amnestorhacus ..................................... 11 andringitra, Caprobolus .......................... 5 Angulifemur ......................................... 10 anichkini, Helicodesmus ..................... 16 annulipes, Ktenostreptus........................ 7 apicomplexus, Hyperbolus .................... 6 Apocoptogonus ...................................... 6 Aporodesmella ....................................... 11 Appalachioria ....................................... 11 Aquattuor ............................................. 6 Arbelorhacus ......................................... 12 Armatostreptus ...................................... 7 armatus, Humbertostreptus (?); typespecies of Armatostreptus ..................... 7 aruba, Giryama ................................... 15 Arvechamboides ....................................... 4
Asiatyla ...................................... 8 asperulus, Gomphodesmus; type-species of Streptelopus ...................................... 21 Aspidiophon ......................................... 10 Aspidiophonini ............................... 10, 11 Asticopyrgodesmus .............................. 12 Atalopharetra ........................................ 12 Atlantodesmus ...................................... 12 atriclavius, Hapsidodesmus ................. 16 Atrophotergum ..................................... 12 attemsi, Helicostreptus ........................... 7

Auliscodesmus ...................................... 12

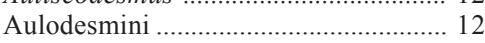

Aulodesmus ........................................... 12

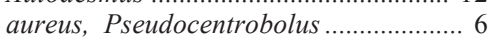
Australocricus ..................................... 5 Austrostrophus ...................................... 5 Aztecolini ……..................................... 5 


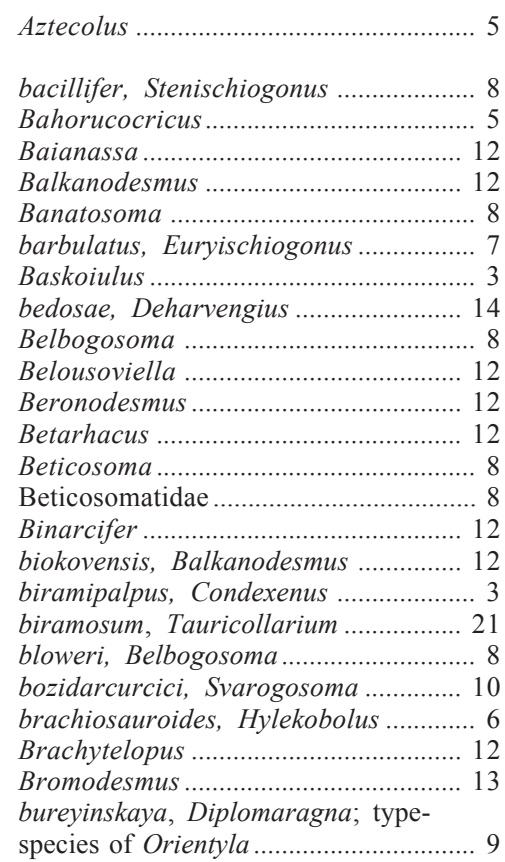

Cacuminostreptus .................................. 7 caecus, Eostemmiulus ........................... 11 callipus, Polydesmus; type-species of Haematotropis ................................ 16 Cambalomma ….................................. 7 Cambalomminae ....................................... 7 cantabrica, Protoglomeris; type-species of Cantabromeris ................................. 3 Cantabromeris ...................................... 3 caoduroi, Osellasoma .......................... 10 Caprobolus ............................................ 5 carbo, Howeosoma .............................. 16 Carinorthomorpha ….......................... 13 Carlocricus ................................................ 5 carminatus, Leptodesmus; type-species of Baianassa ................................... 12 carneus, Elaphogonus ......................... 15 carneus, Polydesmus; type-species of Leptodesmus .......................................... 17 caroli, Limnostreptus .......................... 7 catrionae, Bromodesmus ...................... 13 Causeyella ......................................... 8 cavernicolus, Velebitodesmus ............... 22 Cemsunguria ............................ 2, 13, 20 Centrocladus ......................................... 13 Chelodesmidea ..................................... 13

Chelodesmus ......................................... 13

Chinomorpha .................................... 13

Choristostreptus .................................... 7

circularis, Adisiella ............................. 11

Clastrorhacus ....................................... 13

Clastrotylus .......................................... 13

coelebs, Platyrhacus; type-species of

Trematorhacus ...................................... 21

Colossobolus .............................................. 5

communicans, Gonatodesmus .............. 16 compactilis, Eurydesmus; type-species of Merodesmus ..................................... 18 complexa, Sapamorpha ....................... 20 Condexenus .............................................. 3 conifer, Gomphodesmus; type-species of Virungula .................................... 22 Corallobolus ........................................ 5 Corymborhacus .................................... 13 crassus, Bahorucocricus ...................... 5 crawfordi, Nesoressa ............................ 4 Creagronopus ...................................... 13 crinitum, Taiwaneuma ........................ 10 cristofer, Diplomaragna; type-species of Pacifiosoma ...................................... 10 crucis, Bulgarosoma; type-species of Serbosoma ......................................... 10 cruentus, Corallobolus............................ 5 Crurifarcimen ............................................ 5 Cryptosolenomeris .............................. 13 Curimagua .......................... 2, 13, 14, 22 Curimaguana .................... 2, 13, 14, 22 Cylindromus .................................. 2, 14 Cynotelopus ...................................... 3 Cyrnosoma (invalid genus-group name) .

Cyrnosomatidae (invalid family-group name) ........................................ 8, 9

Dasystigma ........................................... 14 Dazbogosoma .......................................... 9

Deharvengius ........................................ 14

Delirus ......................................... 2, 14

Dendrodesmus ...................................... 14

dendropus, Scoterpes; type-species of

Causeyella ....................................... 8

denticulatus, Aquattuor ........................ 6

derodontus, Sabarhacus ..................... 20

Desmoxytoides ...................................... 14

Diallagmogonini .................................... 14

Diallagomgon ................................... 14

Dibolostethini ............................................. 14

Dibolostethus ....................................... 14

dimorphus, Trigoniulus; type-species

of Parabolus ...................................... 6

Diodontodesminae ................................ 14

Diodontodesmus ....................................... 14

dissimile, Isocladosoma (Centrocladus) 13

divisum, Aspidiophon ........................... 10

Dorasoma ............................................... 3

Dorcadogonus ...................................... 14

doriae, Heterochordeuma ..................... 9

dugopoljica, Massarilatzelia .................. 9

Dysmicodesmus .................................... 14

Ebanodesmus ........................................ 14

echina, Microlympia ............................ 9

Ectopotremia ....................................... 14

eimeri, Leptodesmus (Odontopeltis);

type-species of Atlantodesmus ............ 12

Elaphogonini ........................................ 15

Elaphogonus .................................... 14, 15

elberti, Rhinocricus; type-species of

Carlocricus ........................................... 5

emmrichi, Ngurubates .......................... 18

Emplectomastix ....................................... 15

endemicus, Granitobolus ...................... 5

Enghophyllum ..................................... 4

Eostemmiulus ........................................... 11

Erythranassini ....................................... 15

Erythranassa ........................................... 15

Erythrhacinae ........................................ 15

Erythrhacini ....................................... 15

Erythrhacus ......................................... 15

erythrokrepis, Pachyurus; type-species

of Erythrhacus ................................... 15

Eungellosoma ......................................... 15

Euporogomphus ................................. 15

Euryischiogonus .................................. 7

Euzkadiulus ............................................. 3

everettii, Hoplurorhachis .................... 16

Exaesiotylus ……............................... 15
Exochopyge .......................................... 15

extortus, Ionidesmus ........................... 17

falcata, Sindanina; type-species of

Pseudosundanina ............................... 19

falcatum, Haplogonosoma; type-species

of Simplogonomorpha ......................... 20 falcifera, Brachoria; type-species of Appalachioria .................................. 11 fallens, Platyrrhacus; type-species of Betarhacus ......................................... 12 ferus, Gomphodesmus; type-species of Mitumbagomphus ............................... 18 flagellifer, Monstrodesmus ................. 18 Flagellobolus ........................................... 5 flavipes, Taitastreptus .......................... 8

Gabolus .................................................... 5 gedi, Scaptogonodesmus ..................... 20 Geniculodesmus .................................... 15 Gigantorhacus ...................................... 15

Ginglymodesmus ............................. 15

Giryama .............................................. 15 globusmagicus, Sphaeropoeus; typespecies of Tigridosphaera ................... 4 Gonatodesmus .................................... 16 gounellei, Leptodesmus; type-species of Plectrogonodesmus ......................... 19 Granitobolus

granulata, Curimagua; type-species for both Curimaguana and Vigilia ..... 13, 22 granulatum, Paradoxosoma ............... 19 Graphirhacus ................................... 16 guianensis, Aphelidesmus; type-species of Ochrotropis ................................... 18 Guizhousoma ...................................... 9 Guizhousomatidae ................................ 9

Haematotropis ...................................... 16

Haplogomphodesmus .......................... 16

Hapsidodesmini ...................................... 16

Hapsidodesmus ..................................... 16

hasenpuschorum, Desmoxytoides ........ 14

Helicodesmus .......................................... 16

Helicostreptus .......................................... 7 Helictogomphus ................................... 16 Heptischius .............................................. 7

Heterochordeuma ................................ 9

Heterochordeumatidea .............................. 9 hispaniolus, Rhinocricus; type-species of Quisquellacricus ............................... 6 hoffmani, Mexicopetalum ................... 11

Hoffmanobolidae ...................................... 5 Hoffmanobolus ..................................... 5 Hoffmanorhacus ................................... 16

Hoplurorhachini .................................. 16

Hoplurorhachus ..................................... 16 horvathi, Atropisoma; type-species of Silvattia ............................................. 20 hosei, Hoplurorhachis; type-species of Creagronopus ........................................ 13 Howeosoma ............................................... 16 Hylekobolus ............................................. 6 Hyloecostreptus .................................... 7 Hyperbolus ......................................... 6

iboma, Euporogomphus ...................... 15 Iemanja ............................................ 16 imbecillus, Gomphodesmus; type-species of Molyrogomphus ............................... 18 indigus, Sigodesmus ............................. 20 inexpectata, Nearctomeris ..................... 3 inexpectatus, Habrodesmus; type-species of Geniculodesmus ............................. 15 
Infulathrix ........................................ 9

injucundus, Leonardesmus .................... 17

innupta, Procophorella ......................... 19

insularis, Solentanodesmus ................ 21

intortus, Tomogonus; type-species of

Umbraticus ....................................... 8

Inversispina …….............................. 16

Ionidesmini ..................................... 17

Ionidesmus .................................... 16, 17

Isocladosoma (Centrocladus) ............... 13

Ithynteria ............................................. 17

ivohibiense, Microsphaerotherium ...... 4

jacquelinae, Auliscodesmus .................. 12

jeekeli, Dysmicodesmus ...................... 14

johnsi, Atalopharetra ......................... 12

josianae, Lipseuma ................................ 9

juvenis, Neodesmus ........................... 18

kabaki, Belousoviella ........................... 12

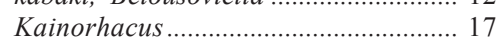

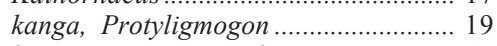

karenae, Masaigomphus ..................... 17

Karukeromus .......................................... 3

khakassica, Shearia ............................ 10

kiellandi, Rondostreptus ........................ 8

Kilimagomphus .................................. 17

Kirkayakidae ................................ 2, 8, 9

Kirkayakus ...................................... 2, 9

koelbeli, Lysiopetalum; type-species of

Schizopetalum ...................................... 11

Koponenius ......................................... 17

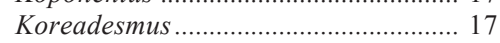

kosciuskovagum, Australiosoma; type-

species of Orocladosoma ..................... 18

krejcae, Sequoiadesmus ........................ 20

Ktenostreptini ........................................ 7

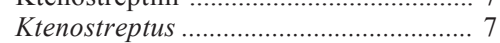

kulla, Notopyrgodesmus ........................ 18

kymatorhabdus, Spirostreptus; type-

species of Namibostreptus .................... 7

lactuca, Heptischius .............................. 7

laeve, Cambalomma ............................ 7

Lamelloramus ......................................... 7

latellai, Guizhousoma .......................... 9

laticlavius, Alluviobolus ....................... 5

lawrencei, Triaenostreptus; type-species

of Choristostreptus ............................... 7

leae, Procyliosoma .................................. 4

leichhardti, Eungellosoma .................. 15

Leodesmus .............................................. 17

leonardi, Retrorsia .............................. 20

Leonardesmus ........................................ 17

Leptodesmidea ........................................ 17

Leptodesmus ......................................... 17

Leschius .................................................. 9

levequei, Trilobodesmus ..................... 21

limbatus, Vanzolegulus ....................... 22

Limnostreptus .................................... 7

Lippus .......................................... 2, 17

Lipseuma ............................................... 9

Lithobiodesmus .................................. 17

Litogonopus ....................................... 17

Lomboknium ....................................... 4

longipenis, Beticosoma ......................... 8

longispina, Schizogomphodesmus ....... 20

luridus, Eurydesmus; type-species of

Clastrotylus

macrodontus, Ulodesmus; type-species of Vaalogomphus ............................... 22 macrogon, Cryptosolenomeris .............. 13 maculosus, Sanguinobolus .................... 6

Madabolus ............................................... 6

magirus, Arbelorhacus ............................ 12

magister, Gabolus .................................. 5

maiala, Asticopyrgodesmus .................. 12

Mammamia ............................................ 4

mandenensis, Riotintobolus .................. 6

mangalisa, Agrophogonus ................... 11

mansuetus, Gomphodesmus; type-species

of Proagomphus ................................... 19

manus, Corymborhacus ..................... 13

margaretae, Lissodesmus; type-species

of Dasystigma .................................. 14

marinezae, Pantanalodesmus ............. 19

Maritimosoma ......................................... 9

marxi, Chelodesmus .............................. 13

Masaigomphus ..................................... 17

Massarilatzelia ..................................... 9

Mastigorhacus ...................................... 18

Mauriesia …......................................... 3

Mauriesiinae ............................................ 3

maximus, Madabolus............................. 6

mazowensis, Cacuminostreptus .............. 7

mcallisteri, Leschius ............................ 9

mecheli, Platyrhacus; type-species of

Kainorhacus ..................................... 17

medius, Tycodesmus ............................. 21

Merodesmini …..................................... 18

Merodesmus ......................................... 18

Metaphora ............................................. 7

mexicanus, Hoffmanobolus .................... 5

Mexicopetalum ...................................... 11

micramma, Ulodesmus ........................ 21

Microlympia .......................................... 9

Microlympiidae ....................................... 9

micromastus, Gomphodesmus; type-

species of Nematogomphus ................. 18

Microsphaerotherium ............................. 4

millequingentesimum, Pagioprium;

type-species of Umbridesmus .............. 21

miniloba, Wulingina ........................... 22

minuta, Carinorthomorpha ................... 13

mirabilis, Emplectomastix .................. 15

mirandus, Platyrhacus; type-species

of Gigantorhacus ................................. 15

Mitumbagomphus ............................... 18

modestus, Dorcadogonus ........................ 14

Molyrogomphus .................................... 18

Monstrodesmus ................................... 18

montana, Chinomorpha ......................... 13

montanus, Spirostreptus; type-species

of Thiangetastreptus ............................. 8

monteithi, Tholerosoma ........................... 21

mossambicus, Polydesmus; type-

species of Aulodesmus ......................... 12

mutara, Litogonopus ........................... 17

naissi, Dazbogosoma ........................... 9 namibiensis, Afraustraloxenodes ........... 3 Namibostreptus ...................................... 7

Nannopetalum ...................................... 9

nasmodes, Stenotyligma ......................... 21

naxius, Brachyiulus (Chromatoiulus);

type-species of Enghophyllum ............... 4

Nearctomeris ......................................... 3

Nematogomphus .................................. 18

Neodesmini ............................................... 18

Neodesmus .............................................. 18

Nesoressa .................................................. 4

Nesoressini ............................................. 4

Nevadesmus ............................................ 18

Ngurubates ............................................ 18 niger, Phaeodesmus; type-species of

Praeterpediculus .................................. 19

nigrior, Spirobolus; type-species of

Aztecolus ............................................... 5

nitens, Harmodesmus; type-species of

Kilimagomphus ..................................... 17

nobile, Sireuma ….................................. 10

Noteremus ......................................... 18

Notopyrgodesmus ................................ 18

obtectus, Parajulus (Pseudojulus) ........ 4

ocala, Arvechamboides ......................... 4

Occitaniulus .......................................... 3

ocellatum, Bulgarosoma; type-species of

Banatosoma ......................................... 8

Ochrotropis ....................................... 18

ologona, Arcidesmus; type-species of

Amnestorhacus ....................................... 11

ophimontis, Nevadesmus ..................... 18

Orientyla .............................................. 9

Orocladosoma ...................................... 18

Orthomorphoides ................................ 19

Osellasoma .......................................... 9, 10

Osellasomatini ...................................... 10

Ostinobolus ............................................ 6

oxylomus, Acerhacus ............................. 11

Pacifiosoma ..................................... 10

paliger, Platyrhacus; type-species of

Tessarorhacus .................................... 21

pallida, Altajella ....................................... 8

pallida, Altajella; type-species of

Kirkayakus ............................................. 9

pallidus, Beronodesmus ............................. 12

pallidus, Setoisenoton .......................... 20

Pantanalodesmus ............................... 19

Parabolus ................................................. 6

Paradoxosoma ...................................... 19

Paradoxosomatidea .............................. 19

Paratylopus ..................................... 2, 17

parcus, Spirobolus; type-species of

Rhinocricus ........................................ 6

Paredrodesmus

parvulus, Gomphodesmus; type-species

of Brachytelopus ................................... 12

pattersonorum, Nannopetalum ............. 9

pauliana, Flagellobolus ........................ 5

pavani, Gomphodesmus; type-species

of Haplogomphodesmus ......................... 16

penicillatus, Apocoptogonus .................. 6

penicillatus, Platyrhacus; type-species

of Graphirhacus ............................... 16

pennsylvanicus, Julus (Julus); type-

species of Ptyoiulus ......................... 4, 5

pensylvanicus (misspelling) .................... 4

Perittogonopus ..................................... 19

perplexa, Pseudoporatia ..................... 19

perplexus, Perittogonopus .................. 19

Perunosoma .......................................... 10

Phyllomatoiulus ...................................... 3

pierrardi, Ectopotremia ........................... 14

planus, Protastrodesmus ..................... 19

Plectrogonodesmus ............................ 19

Pogoro ..................................................... 19

Praeterpediculus ................................. 19

Pratherodesmus ................................. 19

pretorianus, Ulodesmus; type species of

Ithynteria ........................................... 17

Principestreptus ........................................ 3

Proagomphus ........................................ 19

Procophorella ........................................ 19

Procyliosoma ............................................ 4

Procyliosomatidae .................................... 4

profuga, Mammamia ................................. 4 


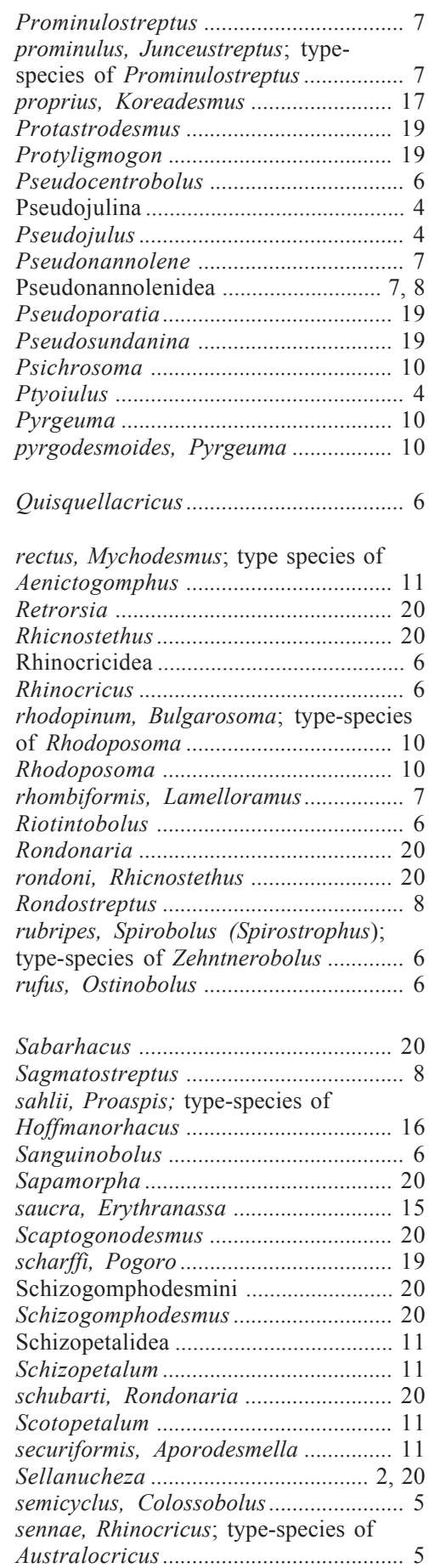

Sequoiadesmus .................................. 20

Serbosoma .............................................. 10

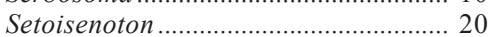

setosa, Orthomorpha; type-species

of Orthomorphoides .............................. 19

setosa, Sinomorpha ............................ 20

Shearia ..... 10

shelleyi, Hyloecostreptus ...................... 7

siam, Infulathrix ................................. 9

sicarius, Dibolostethus ....................... 14

Sigodesmini ......................................... 20

Sigodesmus .......................................... 20

silvaticum, Atrophotergum ................. 12

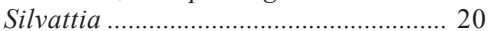

similata, Asiatyla .......................... 8

simplex, Ulodesmus; type-species of

Helictogomphus ................................. 16

simplipodicus, Sinocallipus ................ 11

Simplogonomorpha .............................. 20

Sinocallipodidea ................................... 11

Sinocallipus ........................................ 11

Sinomorpha .......................................... 20

Sireuma ........... 10

Snoqualmia ............................................ 21

snoqualmie, Snoqualmia ..................... 21

Solentanodesmus ......................... 21

solum, Aethalosoma .............................. 11

spatula, Exochopyge .......................... 15

spiliarum, Titanophyllum ....................... 5

spirobolinus, Spirostreptus (Nodopyge);

type-species of Metaphora ..................... 7

splendida, Mauriesia ............................. 3

splendida, Tarbagataya ...................... 10

Stenischiogonus ............................... 8

Stenotyligma ....................................... 21

stictopygus, Austrostrophus ................. 5

stigmatosus, Julus; type-species of

Strongylosoma .................................... 21

Streptelopus ............................................. 21

strongylopygus, Spirostreptus; type-

species of Sagmatostreptus ................... 8

Strongylosomatidea .............................. 21

Strongylosoma ........................................ 21

strongylosomoides, Paratylopus; type-

species of Lippus ................................. 17

summus, Noteremus ................................ 18

superbus, Binarcifer ............................. 12

Svarogosoma ........................................ 10

Szechuanella (under Cemsunguria \&

Sellanucheza) ............................ 2, 13, 20

Taitastreptus ............................................ 8

Taiwaneuma ...................................... 10

Tarbagataya ...................................... 10

tarraconense, Psychrosoma; type-species

of Psichrosoma .................................... 10

tasmanianus, Ginglymodesmus .......... 15

Tauricollarium ................................... 21

taurulus, Paredrodesmus .................... 19
Tectosphaera ............................................ 4

tenebra, Sellanucheza ................... 13, 20 tenebra, Szechuanella; type-species

of Cemsunguria ................................... 13

teresa, Iemanja ...................................... 16

Tessarorhacus ................................ 21

testaceus, Gomphodesmus; type-species

of Diallagmogon.................................. 14

Thiangetastreptus ....................... 8

Tholerosoma ........................................... 21

Tigridosphaera ....................................... 4

Titanophyllum ..................................... 5

Tomogonus .......................................... 2, 8

tortiapicalis, Inversispina ................... 16

Trematorhacus ..................................... 21

tridigitis, Angulifemur......................... 10

Trilobodesmus ...................................... 21

trojanicum, Perunosoma ...................... 10

turova, Diplomaragna; type-species

of Maritimosoma ................................... 9

Tycodesmini ........................................... 21

Tycodesmus ............................................. 21

Tylopus ............................................. 2, 17

typica, Pseudonannolene ...................... 7

Ulodesmini ............................................... 21

Ulodesmus ................................................ 21

Umbraticus .............................................. 2, 8

Umbridesmus ........................................ 21

unicornis, Koponenius ....................... 17

uniporus, Cylindromus; type-species

of Delirus ........................................... 14

Vaalogomphus ................................... 21

vagans, Crurifarcimen .......................... 5

Vanzolegulus ..................................... 22

Velebitodesmus ..................................... 22

vicentae, Tectosphaera ......................... 4

victori, Ebanodesmus ........................... 14

Victoriombrus ....................................... 22

Vigilia ........................................... 2, 22

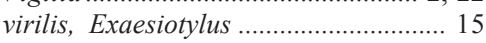

Virungula ............................................. 22

voylesi, Pratherodesmus ..................... 19

warreni, Scolopetalum ......................... 11

woodfordi, Diodontodesmus ............... 14

Wulingina ........................................... 22

xenoporus, Lithobiodesmus ................. 17

yporangae, Alocodesmus; type-species of Leodesmus ......................................... 17 yuma, Dendrodesmus .......................... 14

zehntneri, Siphonophora; type-species of Lomboknium ................................... 4

Zehntnerobolus ..................................... 6

zonatus, Platyrhacus; type-species of

Mastigorhacus .................................. 18 